\title{
Arctic sea ice cover data from spaceborne synthetic aperture radar by deep learning
}

\author{
Yi-Ran Wang and Xiao-Ming Li \\ Key Laboratory of Digital Earth Science, Aerospace Information Research Institute, \\ Chinese Academy of Sciences, Beijing, 100094, China \\ Correspondence: Xiao-Ming Li (lixm@radi.ac.cn)
}

Received: 7 November 2020 - Discussion started: 10 November 2020

Revised: 10 February 2021 - Accepted: 7 May 2021 - Published: 15 June 2021

\begin{abstract}
Widely used sea ice concentration and sea ice cover in polar regions are derived mainly from spaceborne microwave radiometer and scatterometer data, and the typical spatial resolution of these products ranges from several to dozens of kilometers. Due to dramatic changes in polar sea ice, high-resolution sea ice cover data are drawing increasing attention for polar navigation, environmental research, and offshore operations. In this paper, we focused on developing an approach for deriving a high-resolution sea ice cover product for the Arctic using Sentinel-1 (S1) dual-polarization (horizontal-horizontal, HH, and horizontal-vertical, HV) data in extra wide swath (EW) mode. The approach for discriminating sea ice from open water by synthetic aperture radar (SAR) data is based on a modified U-Net architecture, a deep learning network. By employing an integrated stacking model to combine multiple U-Net classifiers with diverse specializations, sea ice segmentation is achieved with superior accuracy over any individual classifier. We applied the proposed approach to over 28000 S1 EW images acquired in 2019 to obtain sea ice cover products in a high spatial resolution of $400 \mathrm{~m}$. The validation by 96 cases of visual interpretation results shows an overall accuracy of $96.10 \%$. The S1-derived sea ice cover was converted to concentration and then compared with Advanced Microwave Scanning Radiometer 2 (AMSR2) sea ice concentration data, showing an average absolute difference of $5.55 \%$ with seasonal fluctuations. A direct comparison with Interactive Multisensor Snow and Ice Mapping System (IMS) daily sea ice cover data achieves an average accuracy of $93.98 \%$. These results show that the developed S1-derived sea ice cover results are comparable to the AMSR and IMS data in terms of overall accuracy but superior to these data in presenting detailed sea ice cover information, particularly in the marginal ice zone (MIZ). Data are available at https://doi.org/10.11922/sciencedb.00273 (Wang and Li, 2020).
\end{abstract}

\section{Introduction}

Sea ice retreat, particularly in the Arctic, has been one of the most significant responses to global climate change (Serreze and Barry, 2011). Therefore, sea ice cover and sea ice concentration are vital parameters for conducting climate change research, navigation in polar regions, and the success of offshore operations.

Spaceborne microwave radiometers have provided the longest time series of sea ice concentration data in polar regions. The large-scale recording of sea ice began with the advent of the Nimbus-5 Electrically Scanning Microwave Radiometer (ESMR) in 1972, followed by the launch of dual- polarization multifrequency systems such as the Nimbus7 Scanning Multichannel Microwave Radiometer (SMMR) (Gloersen et al., 1993) in 1978. Subsequently, the Special Sensor Microwave Imager (SSM/I) multichannel radiometer system (Hollinger et al., 1990) on board the Defense Meteorological Satellite Program (DMSP) satellites and their successor, the Special Sensor Microwave Imager and Sounder (SSMIS) (Kunkee et al., 2008), have provided long timeseries records of sea ice concentration from 1987 to 2016. The typical sea ice concentration data provided by SSM/I and SSMIS have a spatial resolution of approximately $25 \mathrm{~km}$ (Comiso et al., 1997; Parkinson et al., 1999). A new gener- 
ation of passive microwave sensors have been launched in recent decades, mainly represented by the NASA Aqua Advanced Microwave Scanning Radiometer for the Earth Observing System (AMSR-E), which launched in May 2002, followed by the Advanced Microwave Scanning Radiometer 2 (AMSR2) on board the JAXA Global Change Observation Mission-Water (GCOM-W) satellite that launched in May 2012. AMSR-E daily average sea ice concentration products are derived mainly from 19 and $37 \mathrm{GHz}$ channels with the bootstrap technique (Comiso and Sullivan, 1986) with a resolution of $12.5 \mathrm{~km}$. AMSR 2 provides two types of sea ice concentration data: one is based on the bootstrap algorithm (Comiso and Sullivan, 1986) and utilizes vertically polarized brightness temperatures measured from 19 and $37 \mathrm{GHz}$ channels with a resolution of $12.5 \mathrm{~km}$, while the other is based mainly on the ARTIST sea ice (ASI) algorithm (Spreen et al., 2008, 2005) and employs information from a higher frequency of $89 \mathrm{GHz}$, resulting in an increased spatial resolution of $6.25 \mathrm{~km}$. To date, operationally available sea ice concentration data retrieved by spaceborne radiometers have provided reliable measurements of sea ice variations in polar regions. For instance, research shows that the Arctic sea ice extent has decreased since 1978 (Stroeve et al., 2005) and that warming in the Arctic will continue with a rate greater than the current global average (Pachauri et al., 2014).

Due to their large spatial coverage and polar orbits, the microwave radiometers mentioned above are able to acquire daily sea ice concentration information over the Arctic and Antarctic. However, the typical spatial resolution of sea ice data from a spaceborne microwave radiometer ranges from a few to tens of kilometers. Arctic sea ice is experiencing an ongoing rapid decline (Onarheim et al., 2018) and is consequently becoming younger (Nghiem et al., 2007) and thinner (Kwok and Rothrock, 2009) and is drifting faster (Rampal et al., 2009). On the other hand, while there is the retreat of sea ice, the spatial and temporal variations of the Arctic marginal ice zone (MIZ) draws increasing attention (Strong and Rigor, 2013), where the significant interaction between sea ice and ocean dynamics might be an important feedback to sea ice retreat (Thomson and Rogers, 2014). Hence, to better understand sea ice dynamics and its interaction with ocean dynamics (particularly in the MIZ) at different spatial and temporal scales, remote-sensing-based sea ice information with a high spatial resolution is more desirable than ever.

Spaceborne synthetic aperture radar (SAR) has proven to be an ideal remote sensing technique for generating detailed sea ice information because of its inherent capability to image the surface at a high resolution (up to $1 \mathrm{~m}$ to date) independent of sunlight and weather conditions. Moreover, its polarimetric capability enables SAR to have different responses to sea ice types and open water. Since the first civilian SAR instrument, Seasat, was launched in 1978, sea ice monitoring in polar regions has become a primary task of operating spaceborne SAR satellites. Previous studies on sea ice monitoring by spaceborne SAR have focused mainly on the discrimination of sea ice and open water (Hong and Yang, 2018; Komarov and Buehner, 2017), the classification of sea ice types (Wang et al., 2018; Boulze et al., 2020), the detection of icebergs (Power et al., 2001), and the investigation of sea ice drift (Frost et al., 2017). The present study concerns the extraction of sea ice by spaceborne SAR; accordingly, a brief summary of the state-of-the-art SAR-related segmentation techniques for sea ice and open water is given below.

Among the existing SAR-based sea ice segmentation approaches, it is widely agreed that observations acquired under cross-polarization (horizontal-vertical, HV, or verticalhorizontal, $\mathrm{VH}$ ) are more effective than those retrieved under co-polarization (horizontal-horizontal, $\mathrm{HH}$, or verticalvertical, VV) because the former is less sensitive to sea surface backscatter (Dierking, 2013; Scheuchl et al., 2004). However, the strong contrast between sea ice and open water in cross-polarization can be limiting for thin ice with a smooth surface or for open water under strong winds. The difference between co-polarization and cross-polarization data has proven to be an optimal combination for distinguishing between sea ice and open water (Karvonen, 2013; Tan et al., 2018). Recently proposed sea ice segmentation approaches are based mainly on traditional machine learning methods. First, texture features, such as the gray-level cooccurrence matrix (GLCM), energy, correlation, dissimilarity, and entropy, are manually selected and extracted. Then, those texture features are fed into traditional machine learning algorithms such as a support vector machine (SVM) (Zakhvatkina et al., 2017; Leigh et al., 2013; Liu et al., 2014; Li et al., 2020), a random forest algorithm (Tan et al., 2018), or an artificial neural network (Ressel et al., 2015). The performance of traditional machine learning depends heavily on the selection of features. Moreover, although various studies have demonstrated that texture features (such as those based on the GLCM) can effectively reflect the discrepancies between sea ice and open water patterns (Soh and Tsatsoulis, 1999; Clausi and Zhao, 2003; Clausi, 2001), these features fail in cases where sea ice and open water have similar patterns in SAR images (such as an SAR image presenting both windy sea and thin ice surfaces).

The difficulty in designing suitable features for segmentation stems from the complex nature of weather conditions and sea ice states. Hence, manually exhausting all useful texture features for distinguishing sea ice and open water in different situations is challenging. A convolutional neural network $(\mathrm{CNN})$ is a good way to solve this problem. Rather than using hand-crafted features, the $\mathrm{CNN}$ input is the original data (image), and a CNN can automatically and hierarchically learn the features in each network layer. Therefore, the sea ice segmentation model proposed in this study is built upon a particularly successful CNN called U-Net (Ronneberger et al., 2015), which has been widely used in image segmentation and has achieved competitive performance. The novel character of the U-Net architecture provides opportunities for deriving pixelwise sea ice segmentation re- 
sults by high-resolution spaceborne SAR data and with a relatively small number of training samples.

Although various algorithms and methods have been developed for sea ice segmentation by spaceborne SAR data, it seems that valuable SAR-derived sea ice information has not been widely exploited compared with radiometer-retrieved sea ice concentration data, which are routinely utilized for sea ice monitoring in polar regions. This situation may be attributed to two aspects. On the one hand, the existing methodologies have limitations and have seldom been validated with a large dataset. On the other hand, spaceborne SAR data acquisitions over polar regions are often discontinuous due to the limitations of onboard storage or other requested tasks. Alternatively, Sentinel-1A and Sentinel-1B (S1A and S1B, respectively) compose a spaceborne SAR constellation that boasts a significantly shortened revisit frequency of less than $1 \mathrm{~d}$ at high latitudes, e.g., polar regions. Moreover, S1A and S1B have broadly acquired extensive amounts of data in polar regions since their constellation was formed: more than approximately 2500 SAR images in extra wide swath (EW) mode are acquired each month by these two satellites in the Arctic. Therefore, S1A and S1B provide a unique chance to generate high-resolution sea ice information with a high coverage frequency in polar regions.

In this study, we focused on developing a method for deriving high-resolution Arctic sea ice cover information from S1A and S1B based on a deep learning architecture, namely, U-Net. Moreover, aiming for a future operational service, we attempted to generate a standard SAR-derived sea ice cover product by using a full year of S1 images acquired in the Arctic. The remainder of this paper is organized as follows. The datasets used for developing and validating the algorithm are briefly described in Sect. 2. Section 3 presents the proposed sea ice segmentation approach based on the UNet architecture. Following the development of the proposed algorithm, comparisons among SAR-derived sea ice cover data, visual interpretation results, AMSR2 sea ice concentration data, and Interactive Multisensor Snow and Ice Mapping System (IMS) daily sea ice cover data are presented in Sect. 4. Section 5 describes the generated S1-derived Arctic sea ice cover product based on the proposed approach. A discussion and the conclusions are presented in the last two sections.

\section{Datasets}

\subsection{Sentinel-1 data}

To effectively cover the vast Arctic region, S1A and S1B extensively acquire SAR data in EW mode, which has a swath width of approximately $400 \mathrm{~km}$. Moreover, the EW mode data in the Arctic are acquired in dual-polarization $(\mathrm{HH}$ and HV) and are particularly suitable for sea ice monitoring. The S1 EW images used are in ground-range-detected, mediumresolution (GRDM) format with a pixel size of $40 \mathrm{~m} \times 40 \mathrm{~m}$.

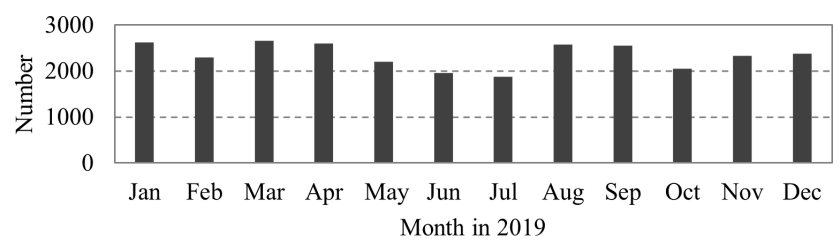

Figure 1. Number of S1 EW images collected in the Arctic region $\left(72.5-83^{\circ} \mathrm{N}\right)$ in each month in 2019.

The incidence angle of the EW data varies from $18.9^{\circ}$ in the near range to $47.0^{\circ}$ in the far range. More than $28000 \mathrm{~S} 1 \mathrm{EW}$ images in dual polarization were collected over the Arctic region $\left(72.5-83^{\circ} \mathrm{N}\right)$ during 2019 . The processing scheme applied to the $\mathrm{S} 1 \mathrm{EW}$ images is described in detail in Sect. 3.1.

\subsection{AMSR2 sea ice concentration data}

The daily AMSR2 sea ice concentration product with a spatial resolution of $6.25 \mathrm{~km}$ released by the University of Bremen was used for a comparison with the S1-derived Arctic sea ice cover data. The AMSR2 sea ice concentration data are retrieved mainly based on the ASI algorithm (Spreen et al., 2008, 2005), which contains an empirical model to retrieve the sea ice concentration and a statistical model of the atmospheric influence. The ASI algorithm mainly uses the difference between the brightness temperatures at $89 \mathrm{GHz}$ under vertical (V) and horizontal $(\mathrm{H})$ polarizations. The $89 \mathrm{GHz}$ channel has the highest resolution among all the channels of the AMSR2 instrument but is more influenced by the atmosphere (e.g., water vapor and cloud liquid water). Thus, the bootstrap algorithm (Comiso and Sullivan, 1986) is employed in conjunction, as it uses the 19 and $37 \mathrm{GHz}$ channels and is therefore less sensitive to atmospheric phenomena (but has a coarser resolution). Accordingly, the S1-derived sea ice cover data were first converted into sea ice concentration data on a regular grid of $6.25 \mathrm{~km}$ and then matched with the AMSR2 sea ice concentration data on a pixel-by-pixel basis.

\subsection{IMS sea ice cover data}

Another reference dataset, the sea ice cover data from the IMS (https://nsidc.org/data/G02156/versions/1, last access: 10 June 2021) released by the National Snow and Ice Data Center (NSIDC), was employed for a comparison with the S1-derived Arctic sea ice cover data. The data are considered valid at 00:00 UTC each day. To determine the presence of sea ice, visible imagery is first retained when not obstructed by clouds; then, passive microwave data and the National Ice Center (NIC) weekly sea ice analysis product are applied depending on the time of year, resolution, and data latency (Ramsay, 1998). In this study, IMS data at a spatial resolution of $1 \mathrm{~km}$ are used. As both sources of data record sea ice cover information, the IMS data and S1-derived data were compared directly in corresponding pixels. 


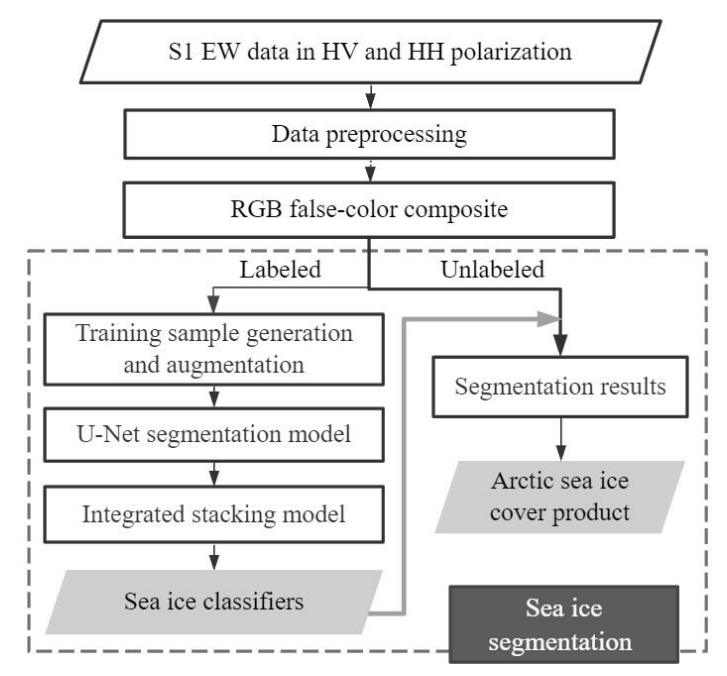

Figure 2. Flowchart of the proposed method for deriving sea ice cover information from S1 EW images in $\mathrm{HV}$ and $\mathrm{HH}$ polarization.

\section{Methodology}

\subsection{Overall S1-based sea ice segmentation approach}

The overall architecture of the proposed approach for deriving sea ice cover from S1 EW images in HV and HH polarization is depicted in Fig. 2. First, the S1 EW images are preprocessed, which includes radiometric calibration, denoising, incidence angle correction, resampling, and land masking. Then, the preprocessed HV- and HH-polarized data are synthesized into an RGB false-color composite, which serves as the input for the proposed sea ice segmentation model. During the training process, a small proportion of the RGB false-color composites is labeled as training data and fed into the U-Net segmentation model, generating well-trained sea ice classifiers. The integrated stacking model combines the classifiers from the U-Net model to generate an aggregate sea ice classifier. The aggregate classifier is then applied to all other unlabeled data to generate Arctic sea ice cover data.

\subsection{S1 image preprocessing}

S1A and S1B EW data in HV and HH polarization were used to develop the proposed algorithm and generate the Arctic sea ice cover product.

The radar backscatter of S1 EW images in HH polarization changes rapidly with variation in the incidence angle. Thus, prior to using these data to derive sea ice cover information, all the EW data in $\mathrm{HH}$ polarization are processed for an incidence angle correction. The linear regression method for the $\mathrm{HH}$ backscatter versus elevation angle introduced in Murashkin et al. (2018) is used. Figure 3a and b show an example of an S1 EW HH-polarized image before and after an incidence angle correction. In contrast, the HV-polarized image does not reveal a significant sensitivity to the inci- dence angle; therefore, the incidence angle correction is not applied.

The S1 EW images in HV polarization are strongly affected by the scalloping effect and thermal noise. Thus, all the EW data in HV polarization are denoised using the method proposed in Sun and Li (2020). This denoising method improved upon previous methods (Park et al., 2017) by segmenting the image into more azimuthal blocks and introducing a variance factor to discriminate homogeneous and inhomogeneous blocks, thereby deriving a more accurate scaling factor and balancing factor. In addition, we proposed a new method in Sun and Li (2020) for eliminating residual noise (i.e., multiplicative noise) at the sub-swath boundaries of EW data. Figure 3c and d show an example of an S1 EW $\mathrm{HV}$-polarized image before and after denoising, demonstrating good performance in removing both additive and multiplicative noise present in the EW HV-polarized image.

As the original S1 EW images are quite large, these data are downsampled to achieve a more manageable file size to be handled by the sea ice segmentation model. The denoised and calibrated S1 images are averaged by a $10 \times 10$ window, resulting in a change in pixel size from $40 \mathrm{~m}$ to $400 \mathrm{~m}$. Thus, the pixel-based sea ice segmentation results also have a pixel size of $400 \mathrm{~m}$. The land area is masked as black in the images by the Global Self-consistent Hierarchical High-resolution Geography Database (GSHHG; https://www.ngdc.noaa.gov/ $\mathrm{mgg} / \mathrm{shorelines/gshhs.html,} \mathrm{last} \mathrm{access:} 10$ June 2021) with the full grid resolution of $1 \times 1 \mathrm{arcmin}$.

\subsection{Combination of S1 co- and cross-polarization data}

For sea ice segmentation by spaceborne SAR data, the radar backscatter intensity naturally constitutes the basis of the determination. Many studies consider the cross-polarization channel to be very effective for sea ice detection because it is sensitive to ice-induced volume scattering, while the sea surface generally presents surface scattering. Thus, in the crosspolarization channel, the radar backscatter of sea ice is generally higher than that of open water. However, this is not always the case. Under strong winds, open water can present radar backscatter intensities comparable to those of sea ice (an example is shown in Fig. 4b, where the sea surface wind speed varies between approximately 8 and $18 \mathrm{~m} \mathrm{~s}^{-1}$ according to the ERA5 reanalysis wind field at synoptic time; Hersbach et al., 2020). Moreover, sea ice with a smooth surface can have a low backscatter intensity (Aldenhoff et al., 2019) (see Fig. $4 \mathrm{~d}$ for example). These substantial variations in the SAR radar backscatter intensities of sea ice and open water make sea ice segmentation a challenging task. As mentioned in the introduction, the difference between co-polarization and cross-polarization data on sea ice and open water is also effective for distinguishing between sea ice and open water. Therefore, in addition to incorporating cross-polarization data directly, we adopted polarization ratio $(\mathrm{HH} / \mathrm{HV})$ and polarization difference $(\mathrm{HH}-\mathrm{HV})$ data in the proposed ap- 


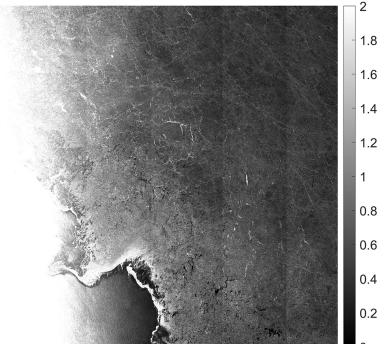

(a)

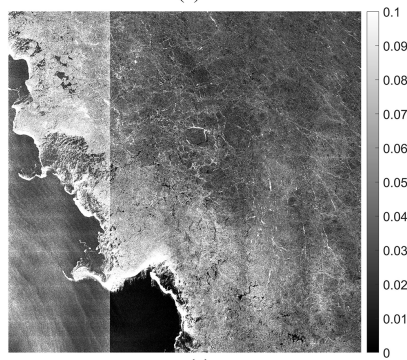

(c)

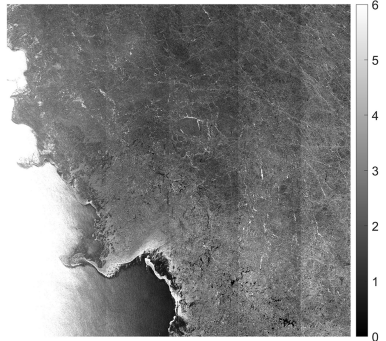

(b)

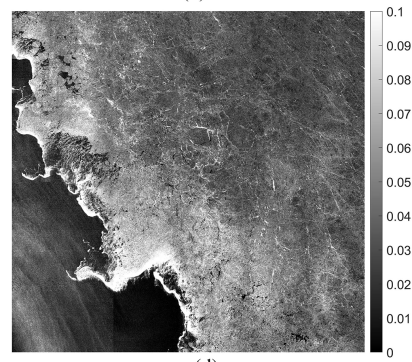

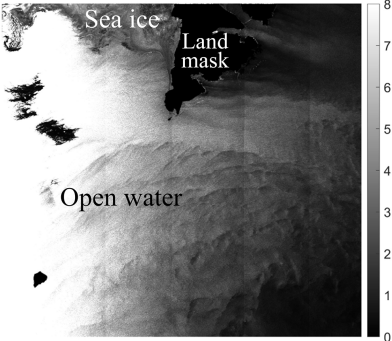

(a)

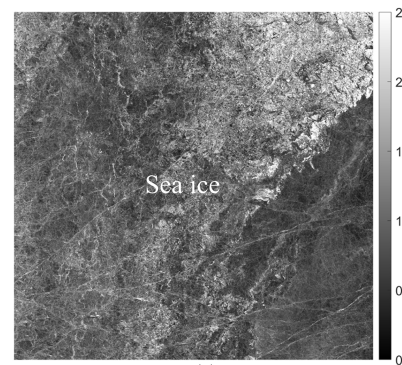

(c)

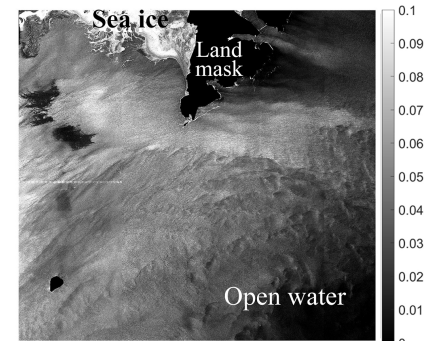

(b)

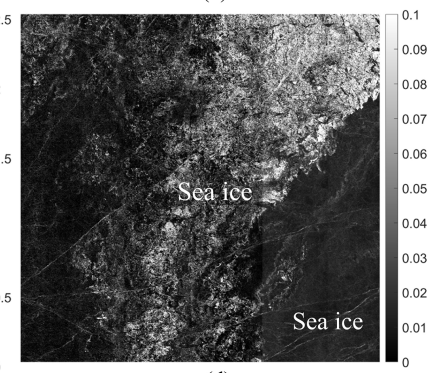

(d)
Figure 3. Example of the denoising process. (a) The original S1 EW HH-polarized image. (b) S1 EW HH-polarized image after an incidence angle correction. (c) The original S1 EW HV-polarized image. (d) Denoised S1 EW HV-polarized image using the denoising method (Li, 2020). (Image ID: S1B_EW_GRDM_1SDH_20180711T073522_ 20180711T073622_011760_015A2A_4965).

proach, which has been proved to be useful for sea ice discrimination and other feature extraction from SAR data (e.g., Dong et al., 2012; Murashkin et al., 2018).

Figure 5 illustrates the process of combining the HVpolarized data with the polarization ratio and polarization difference data into an RGB false-color composite, which serves as the input in the sea ice segmentation process. For the HV-polarized S1 images, the radar backscatter in linear units is scaled to $0-255$ after discarding $2 \%$ of the maximum and minimum values each. Then, each image is scaled according to its data range to maximize the texture features. The $\mathrm{HH} / \mathrm{HV}$ and $\mathrm{HH}-\mathrm{HV}$ data are first converted into decibel units and then stretched to fixed thresholds of $[2 \mathrm{~dB}$, $7 \mathrm{~dB}]$ and $[-2 \mathrm{~dB}, 3.5 \mathrm{~dB}]$, respectively, to keep the absolute difference between the HV- and HH-polarized data. These thresholds ensure approximately $95 \%$ of the $\mathrm{HH} / \mathrm{HV}$ and $\mathrm{HH}-\mathrm{HV}$ values falling into the range, which were determined according to the statistics of more than 200 S1 EW images acquired under different scenarios of sea ice and open water. The polarization ratio or polarization difference values beyond these two ranges are replaced by the corresponding thresholds, and then a linear stretch to $0-255$ is applied. Finally, the scaled HV-polarized data, polarization difference data, and polarization ratio data serve as the red, green, and blue channels, respectively, to synthesize an RGB false-color composite image. The RGB false-color composite clearly presents more textures and details than either the
Figure 4. Examples of S1 EW images presenting the challenges in sea ice segmentation. (a) HH-polarized and (b) HV-polarized S1 EW images of a windy sea surface. (c) HH-polarized and (d) HVpolarized S1 EW images of a smooth thin ice surface. (Image ID: a and b S1A_EW_GRDM_1SDH_20190130T060740_ 20190130T060840_025703_02DB20_85BC; c and d S1B_EW_ GRDM_1SDH_20190115T194015_20190115T194115_ 014509_01B066_11F1).

HV-polarized image or the HH-polarized image and therefore lays a better foundation for further sea ice segmentation tasks.

\subsection{Generation of training samples}

Precisely labeled samples are necessary for the successful development of machine learning algorithms. We first discriminated sea ice and open water by using $251 \mathrm{~S} 1 \mathrm{EW}$ images based on our previously developed SVM classification ( $\mathrm{Li}$ et al., 2020), from which the good results (judged by visual inspection) were further used as initial training data. Then, manual checking was performed to improve the correctness and completeness of the classification results. The selection of the training data considered both the data acquisition location (see Fig. 6a) and the data acquisition season (see Fig. 6b) to represent diverse sea ice conditions. Figure $6 \mathrm{c}$ shows examples of four RGB false-color composite images and their corresponding precisely labeled data, in which sea ice is labeled 1 (white) and open water is labeled 0 (black).

We took two thirds of the total 251 labeled S1 EW images as the training dataset, and we used the remaining one third of the samples as the evaluation dataset. The labeled dataset is further grouped for the integrated stacking model, which is described in a later section. To fit the network, first, the labeled data are cropped into patches with dimensions 


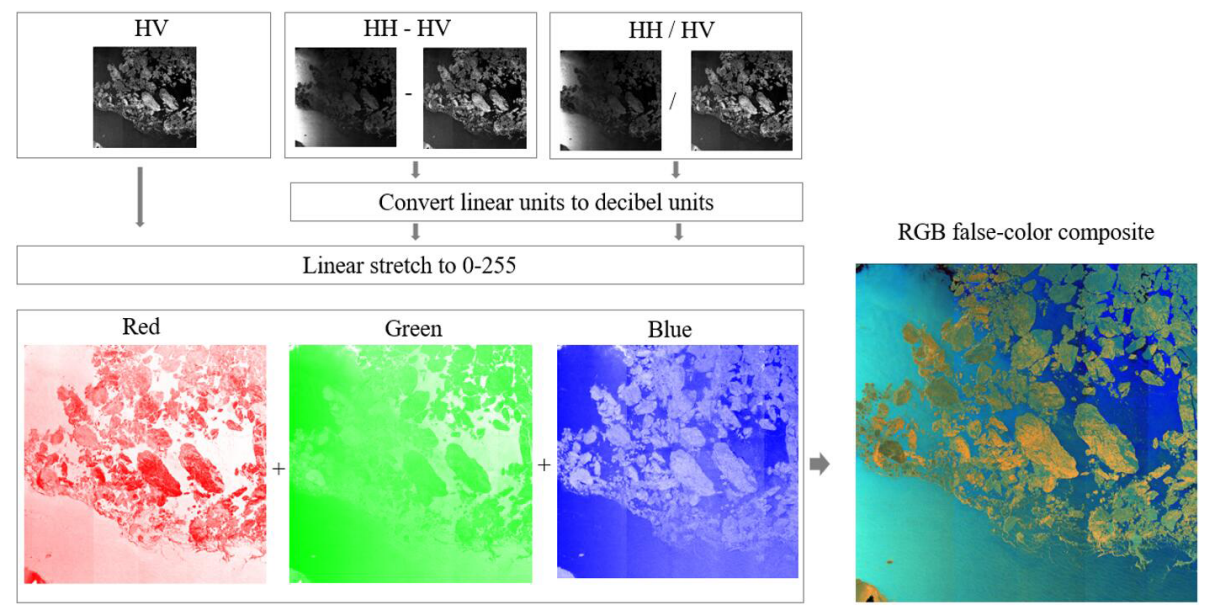

Figure 5. Illustration of combining HV-polarized data with polarization ratio and polarization difference data into an RGB false-color composite, which presents more textures and details than either HV- or HH-polarized images.

of $256 \times 256$ pixels. The patch size of $256 \times 256$ pixels is an empirical value weighed between manageable model size and sufficient information one patch has. Then, training sample augmentation is performed for each patch by rotating and flipping the image, and the expanded dataset is further processed by adjusting the hue (as illustrated in Fig. 6d) to generate additional samples. The augmentation process dramatically increases the quantity and diversity of the training samples, which improves the performance of the deep learning model and reduces overfitting. The evaluation dataset is also cropped, but augmentation is not performed. After patch extraction and training sample augmentation, the total amount of training and evaluation samples reaches more than 8000 .

\subsection{Modified U-Net}

The overall architecture of the proposed sea ice segmentation model is shown in Fig. 7. U-Net is particularly good at sea ice segmentation in SAR images for the following two reasons. (1) The network can perform localization effectively to provide high-resolution segmentation masks by labeling each pixel of the input image with a corresponding class, i.e., sea ice or open water. (2) The network works well with small datasets and is relatively robust against overfitting, although obtaining a large number of labeled samples from remote sensing data is challenging.

The architecture of the sea ice segmentation model contains two parts, i.e., an encoder part and a decoder part. The encoder, which captures the discriminative features in the image, is composed of a stack of convolutional layers, batch norm layers, and rectified linear unit (ReLU) operations, followed by a max pooling layer, where each max pooling layer reduces the spatial resolution of the input layers by a factor of 2 . Then, the batch norm layers are added to each of the blocks to increase the learning speed. The decoder part, which semantically projects the discriminative features learned by the encoder onto the pixel level, consists of upsampling and concatenation operations, followed by convolutional layers. To obtain pixel-based segmentation results, at every decoder step, we used skip connections by concatenating the output of the transposed convolutional layers with the feature maps from the encoder at the same level. High-resolution features from the contracting layers are combined with the upsampled output; hence, these layers increase the resolution of the output. A final convolutional operation with a kernel size of $(1,1)$ and a sigmoid activation function is performed at the output side of the network. The network outputs the ultimate segmentation mask in terms of a $256 \times 256$ matrix (same size as the input) with values between 0 and 1 . In the segmentation mask, the closer a pixel value is to 1 , the more likely it is sea ice, and vice versa for water.

The network is trained using Adam optimization (Kingma and $\mathrm{Ba}, 2014)$ with a batch size of eight. In our case, the performance of the model improves with the increasing of the batch size. However, due to the limitation of the memory capacity, the batch size of eight is the maximum value the device can handle. A specialized segmentation loss function that combines binary cross-entropy and dice loss is chosen to evaluate the final results as

$H_{p}(q)=-\frac{1}{N} \sum_{i=1}^{N} y_{i} \log \left(p\left(y_{i}\right)\right)+\left(1-y_{i}\right) \log \left(1-p\left(y_{i}\right)\right)$,

where $y$ is the label ( 1 for sea ice and 0 for water) and $p(y)$ is the predicted probability of the pixel being sea ice for all $N$ pixels in each image. A perfect model would have a performance of $H_{p}(q)=0$.

The input RGB false-color composite is cropped into patches of $256 \times 256$ pixels and then fed into the model. Predictions made for these patches by the sea ice segmentation model are regrouped accordingly. Neighboring patches have 


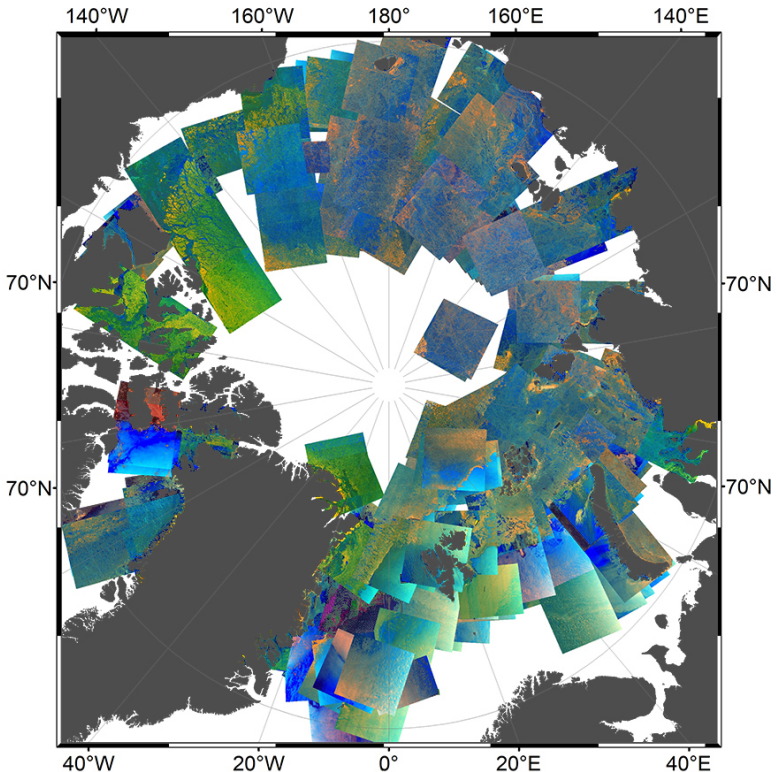

(a)

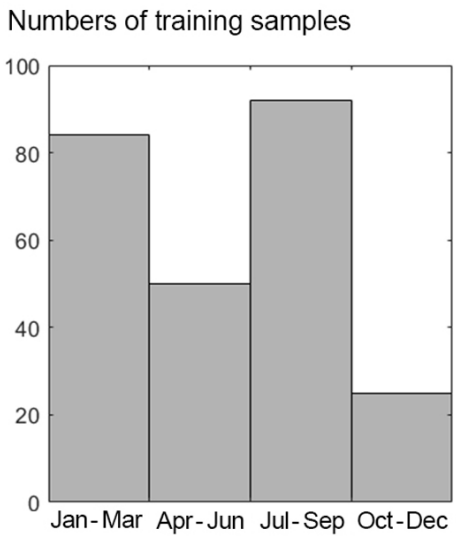

(b)
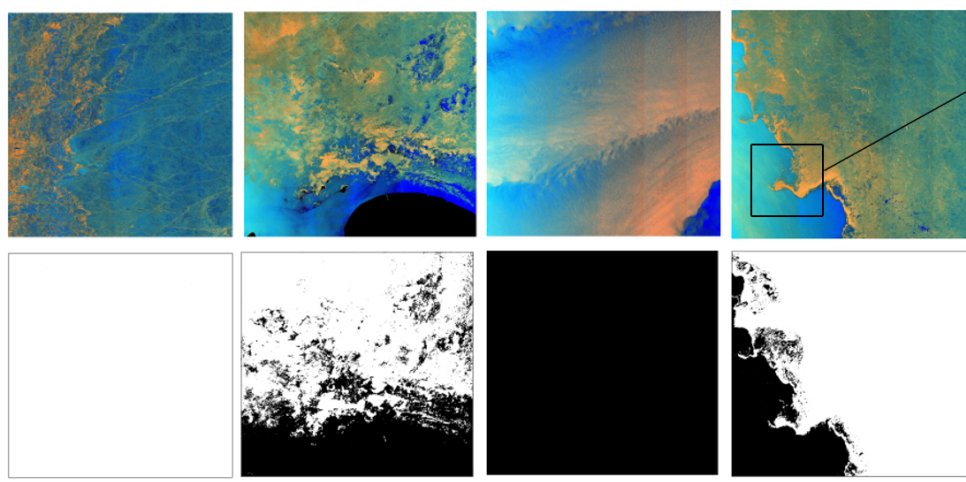

(c)

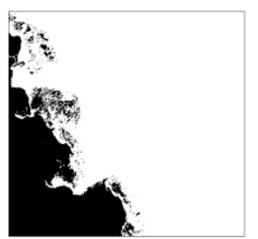

(20)

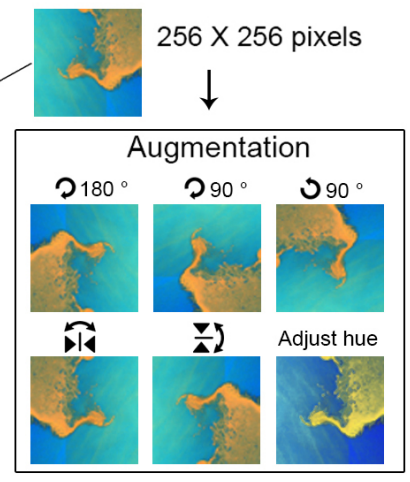

(d)

Figure 6. (a) Spatial distribution of the $\mathrm{S} 1$ images used for training the sea ice segmentation model. The images are shown as RGB false-color composites, and the land is masked in gray. (b) Histogram of the seasonal distribution of the training data. (c) Examples of data labeling: the top row shows four RGB false-color composite images, and the corresponding labeled images are in the bottom row. (d) Illustration of the augmentation process to increase the quantity and diversity of the samples in the training dataset.

an overlap of 12 pixels to reduce artifacts along the edges of the patches.

\subsection{Integrated stacking model}

During the training and validation of the U-Net model, it is almost impossible to find a perfect model for all scenarios. For example, one model specializes in detecting continuous and large areas of sea ice but tends to output biased segmentation results for windy sea surfaces (e.g., examples shown in Fig. 4a and b), while another model performs well at segmenting regions with highly mixed sea ice and open water (particularly over the MIZ) but performs poorly at containing thin ice with a smooth surface (e.g., examples shown in Fig. 4c and d). This is understandable, as Wolpert and Macready (1997) demonstrated in their "no free lunch" the- orem, because an algorithm that performs well in one class of problems must "pay" for that accuracy with degraded performance on a set of all remaining issues.

The performance of each model diverges upon being fed a different set of training data. We divided the training samples into groups and trained the U-Net model with different datasets, thereby generating several classifiers. In our sea ice segmentation approach, we adopted the idea of stacked generalization (Wolpert, 1992) to enable each model to fully utilize its strengths and mitigate its weaknesses, resulting in higher accuracy and sensitivity. Stacked generalization, or "stacking", is an ensemble machine learning algorithm that involves combining the outputs of several networks into an aggregate output, which often improves the accuracy over any individual output. 


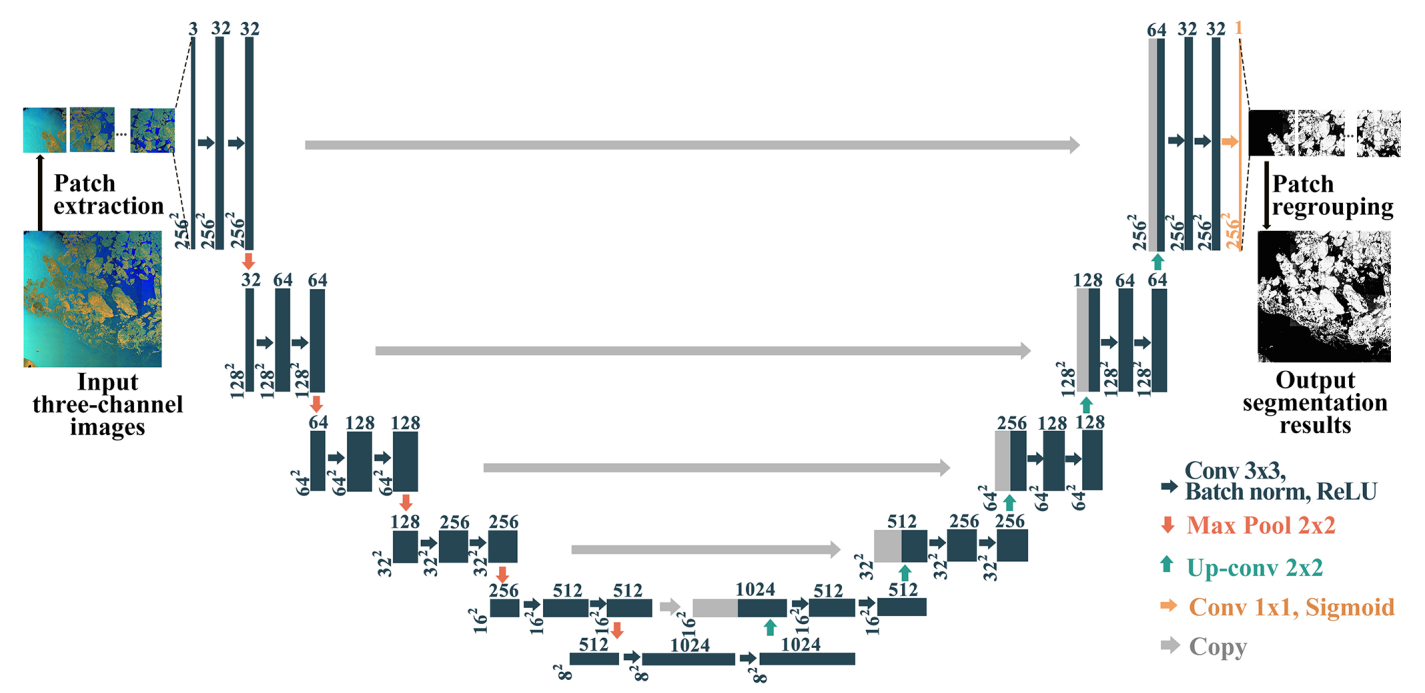

Figure 7. The overall architecture of the proposed sea ice segmentation model for the S1 EW data based on the U-Net deep learning algorithm.

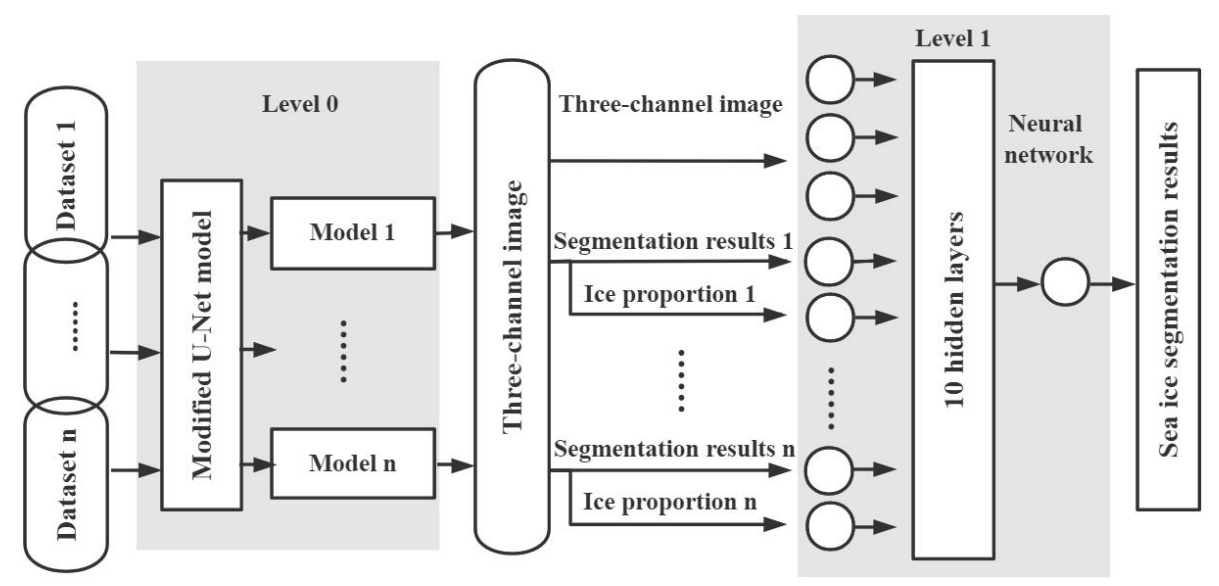

Figure 8. Overall architecture of the proposed integrated stacking model for generating the S1-derived sea ice cover data.

As illustrated in Fig. 8, the architecture of the proposed integrated stacking model consists of two levels. The first level, i.e., level 0 , is formed by base classifiers. Five U-Net classifiers with diverse specializations are selected as the base classifiers of level 0 . Figure 9 shows several cases to give a more visual representation of the selected classifiers and the integrated stacking model. The first two columns contain S1 images in HV polarization and the corresponding RGB falsecolor composites. The following five columns are the outputs from the selected five classifiers with diverse specializations presented in grayscale with white for 1 and black for 0: the closer the number is to 1 , the more likely it is sea ice, and vice versa for water. Notably, models 2 and 3 are specialized for large areas of sea ice, especially newly formed sea ice, whereas models 1 and 4 produce fewer wrong segmentation results for high wind sea surfaces, and models 3 and 5 deliver more details when ice floes are mixed with water.
The five classifiers are then applied to all the labeled datasets. The output segmentation results, together with the four RGB false-color composite images, are then used as the input data for level 1 . We found that the performance of each classifier in level 0 is highly correlated with the percentage of sea ice in one S1 EW image; thus, the sea ice proportion is calculated and added to the inputs for level 1 . The process of integrated stacking can be considered as several classifiers vote for the final segmentation. The classifier will have higher voting weights in their specialized scenarios. The scenarios are described by ice cover proportion to the full coverage of a S1 EW scene, which is one of the inputs for our integrated stacking model. For instance, for the scenarios with high ice proportion, models 2 and 3 will have greater voices. The weights allocation is realized by a neural network containing 10 hidden layers that combine the outputs of the base models, marked as level 1 in Fig. 8. Note that level 0 and level 


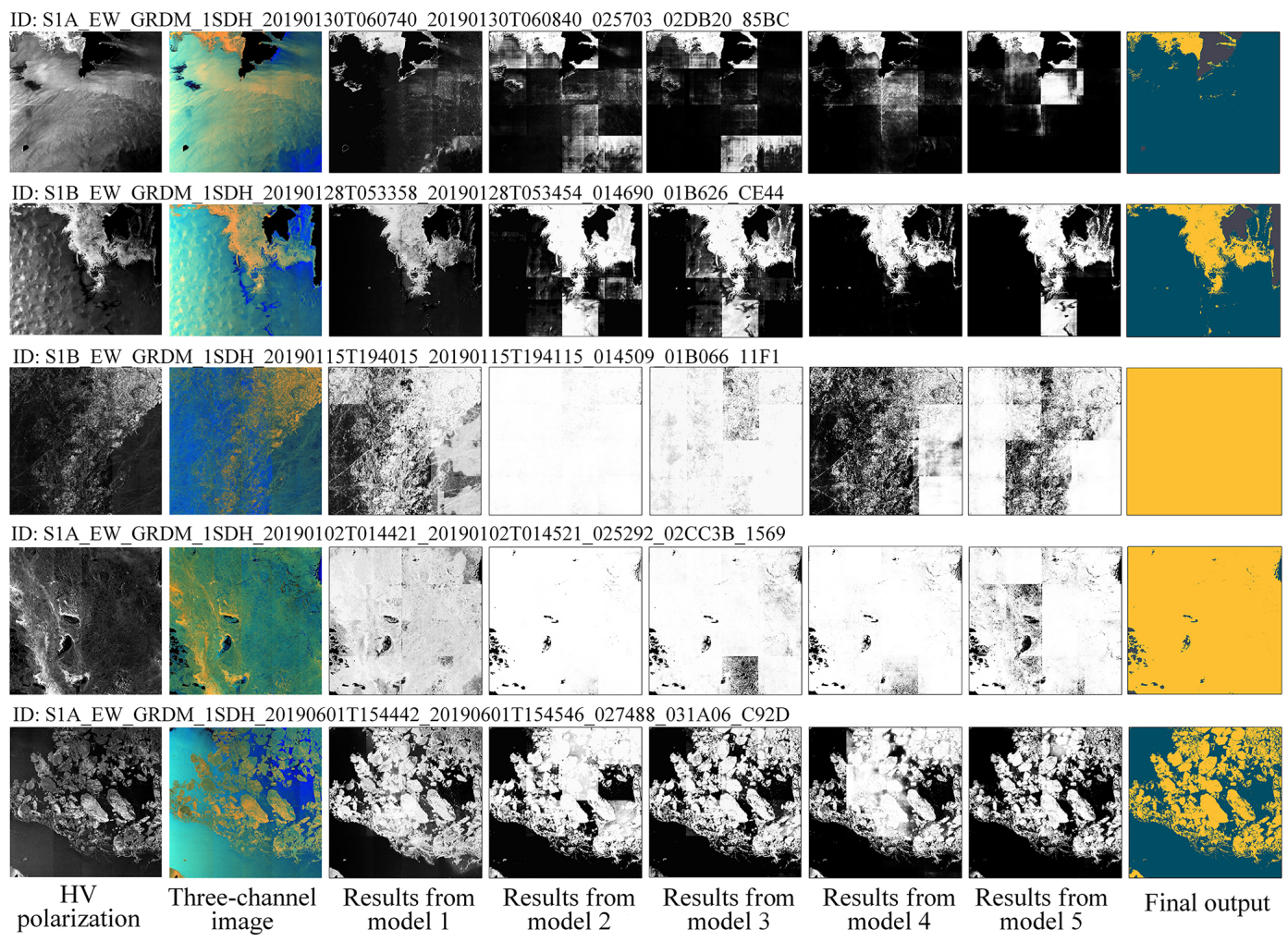

Figure 9. Five cases illustrating the combination of selected U-Net classifiers into the integrated stacking model. The first two columns are S1 images in HV polarization and the corresponding input RGB false-color composites. The following five columns are the outputs from the five classifiers selected with diverse specializations, and the last column is the final output of sea ice cover (yellow, cyan, and gray represent sea ice, open water, and land, respectively).

1 use the same training set; however, the dataset in level 0 is divided into several subsets for a different classifier, but in level 1, all the data are fed into the neural network. For the final output of the integrated stacking model, a value of 0.5 is taken as the threshold to judge whether a pixel is classified as 0 (open water) or 1 (sea ice), as shown in the last column of Fig. 9, with yellow representing sea ice, cyan representing open water, and gray representing land.

\section{Results}

We applied the developed U-Net-based sea ice segmentation model to over 28000 S1 EW images acquired in the Arctic in 2019 to obtain sea ice cover data at a spatial resolution of $400 \mathrm{~m}$. Those S1-derived sea ice cover data are compared with visual interpretation results, AMSR2 sea ice concentration data, and IMS sea ice cover data.

\subsection{Comparison with visual interpretation results}

We conducted a comparison between the S1-derived Arctic sea ice cover data and the pixel-level visual interpretation results based on 96 cases. The selection of the cases for comparison considers the situations of sea ice in different sea- sons and is as objective as possible. The $28000 \mathrm{~S} 1$ images are roughly divided into four groups based on sea ice proportion $(0 \%-25 \%, 25 \%-50 \%, 50 \%-75 \%, 75 \%-100 \%)$ according to the derived sea ice cover; then we select one S1A and one S1B image in each of the four groups with the earliest acquisition time in each month in 2019. Thus, in total $4 \times 12 \times 2=96$ cases were selected and manually interpreted for segmentation of sea ice and open water.

Figure 10 shows an example of the S1-derived sea ice cover, the visual interpretation, and a subsequent comparison. This image was acquired at the northeast of Severnaya Zemlya, presenting large areas of both open water and floating sea ice. Figure 10a shows the RGB false-color composite image. Figure $10 \mathrm{~b}$ is the corresponding S1-derived sea ice cover result. Figure 10c presents the visual interpretation of sea ice and open water segmentation. Figure 10d shows the differences between S1-derived and visual interpretation results, suggesting a good agreement between them. Some subtle differences exist around the boundary of the sea ice, which is confusing even for human eyes.

For a quantitative comparison, the proportions of true positive (TP, correctly classified sea ice), true negative (TN, correctly classified open water), false positive (FP, incorrectly classified sea ice), and false negative (FN, incorrectly clas- 


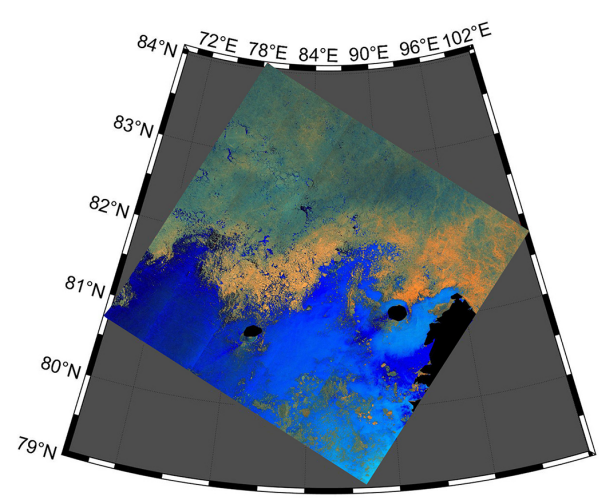

(a)

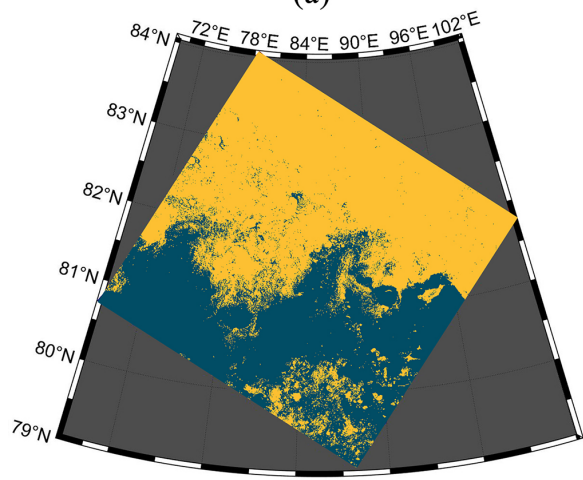

(c)

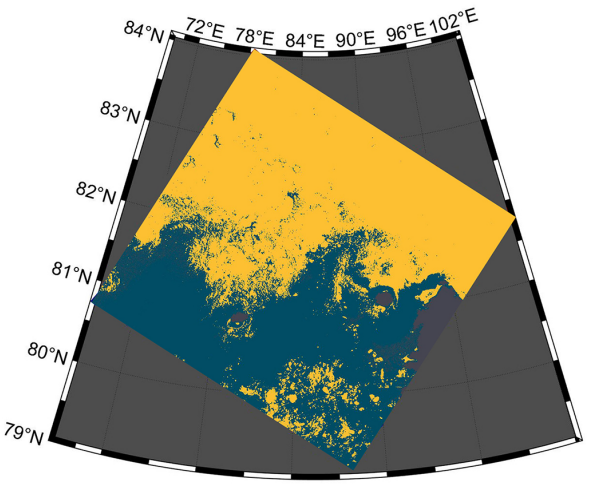

(b)

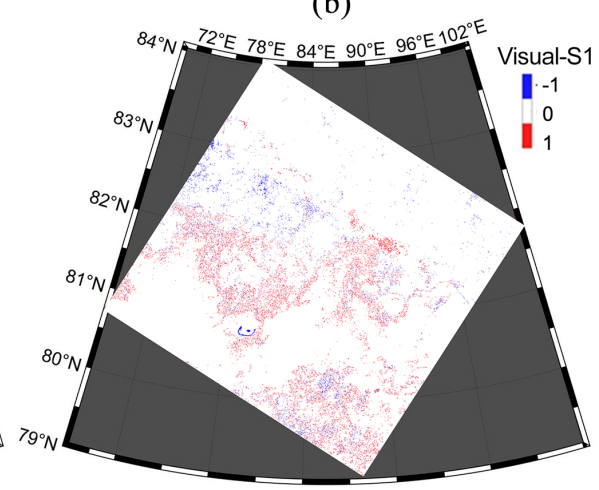

(d)

Figure 10. An example of S1-derived sea ice cover and its comparison with the visual interpretation result. (a) RGB false-color composite image. Panels (b) and (c) are S1-derived and visual interpretation of sea ice cover, with yellow indicating sea ice, cyan indicating open water, and gray indicating land. (d) Differences in sea ice cover between (b) and (c) (visual interpretation-S1 derive). (Image ID: S1B_EW_GRDM_1SDH_20190801T022310_20190801T022410_017386_020B16_F790).

sified open water) of the 96 cases are listed in Table 1. The overall performance is evaluated by the accuracy, i.e., Accuracy $=\left(n_{\mathrm{TP}}+n_{\mathrm{TN}}\right) \times 100 \% / n_{\text {total }}$, where $n_{\mathrm{TP}}$ and $n_{\mathrm{TN}}$ are the pixel numbers of TP and TN, and $n_{\text {total }}$ denotes the total pixels of the derived result. For the cases with a sea ice proportion of $25 \%-75 \%$, the accuracy of $91.61 \%$ is relatively lower than the accuracy of other groups due to highly complex situations of sea ice and open water mixing, while for the cases with an overwhelming majority of sea ice (group 4) or open water (group 2), the accuracy can be as high as approximately $99 \%$. The overall accuracy is $96.10 \%$ for all the 96 cases.

As the pixel-level visual interpretation is time-consuming, it is not possible to evaluate the S1-derived sea ice cover for a large amount of data by comparing it with visual interpretation. We then used the AMSR2 sea ice concentration data and IMS sea ice cover data with relatively low spatial resolution for further comparison.
Table 1. Proportions of TP, FP, TN, FN, and accuracy of the S1derived sea ice cover data evaluated by visual interpretation results of 96 cases.

\begin{tabular}{lrrrrr}
\hline $\begin{array}{l}\text { Sea ice } \\
\text { proportion }\end{array}$ & TP & FP & TN & FN & Accuracy \\
\hline $0-25 \%$ & $11.19 \%$ & $0.94 \%$ & $87.23 \%$ & $0.64 \%$ & $98.42 \%$ \\
$25 \%-50 \%$ & $32.82 \%$ & $2.45 \%$ & $62.15 \%$ & $2.58 \%$ & $94.97 \%$ \\
$50 \%-75 \%$ & $55.75 \%$ & $3.38 \%$ & $35.87 \%$ & $5.01 \%$ & $91.61 \%$ \\
$75 \%-100 \%$ & $96.59 \%$ & $0.27 \%$ & $2.59 \%$ & $0.55 \%$ & $99.19 \%$ \\
\hline Overall & $49.73 \%$ & $1.74 \%$ & $46.36 \%$ & $2.17 \%$ & $96.10 \%$ \\
\hline
\end{tabular}

\subsection{Comparison with the AMSR2 sea ice concentration data}

We conducted a comparison between the S1-derived Arctic sea ice cover data and the AMSR2 sea ice concentration data. The S1-derived sea ice cover data (with a spatial resolution of $400 \mathrm{~m}$ ) were converted into sea ice concentration on a regular grid of $6.25 \mathrm{~km}$ (the same as the spatial resolution of the AMSR2 data), with the center of each grid corresponding to each pixel of AMSR2. Then, the sea ice concentration data were matched with the AMSR2 data on a pixel-by-pixel ba- 


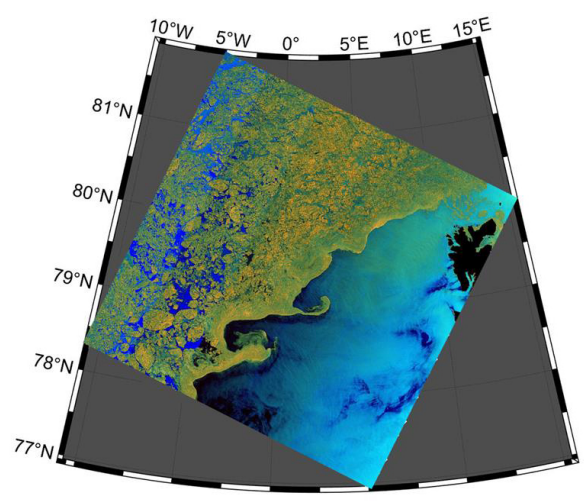

(a)

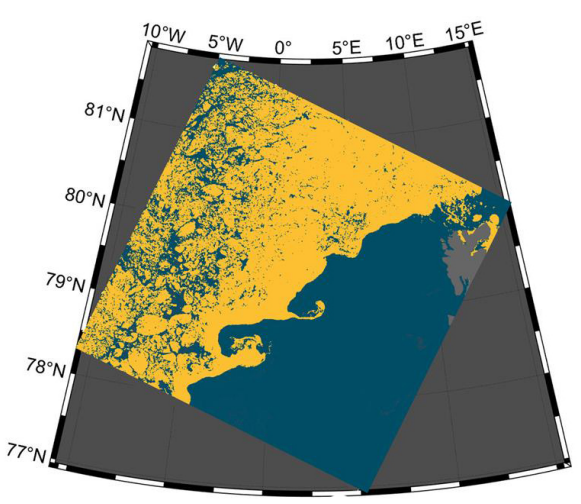

(b)

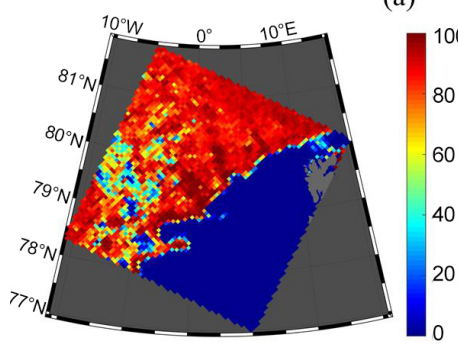

(c)

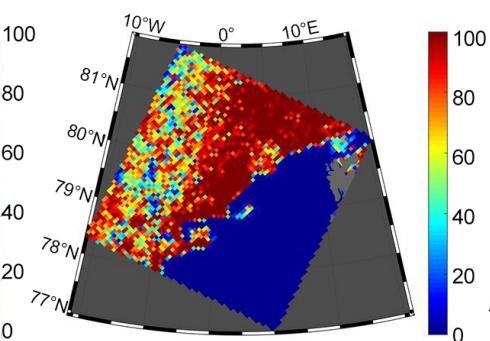

(d)

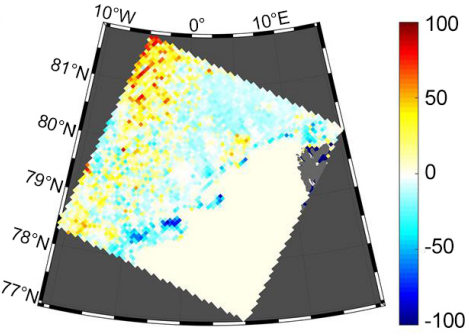

(e)

Figure 11. Example of S1-derived sea ice cover data and the comparison with the AMSR2 data. (a) RGB false-color composite image. (b) S1-derived sea ice cover result. Yellow indicates sea ice, cyan indicates open water, and gray indicates land. (c) Sea ice concentration based on the S1-derived result. (d) AMSR2 sea ice concentration. (e) Differences in the sea ice concentration between the AMSR2 data and S1-derived results (AMSR2-S1). (Image ID: S1A_EW_GRDM_1SDH_20190601T072854_20190601T072958_027483_0319D7_E0F1).

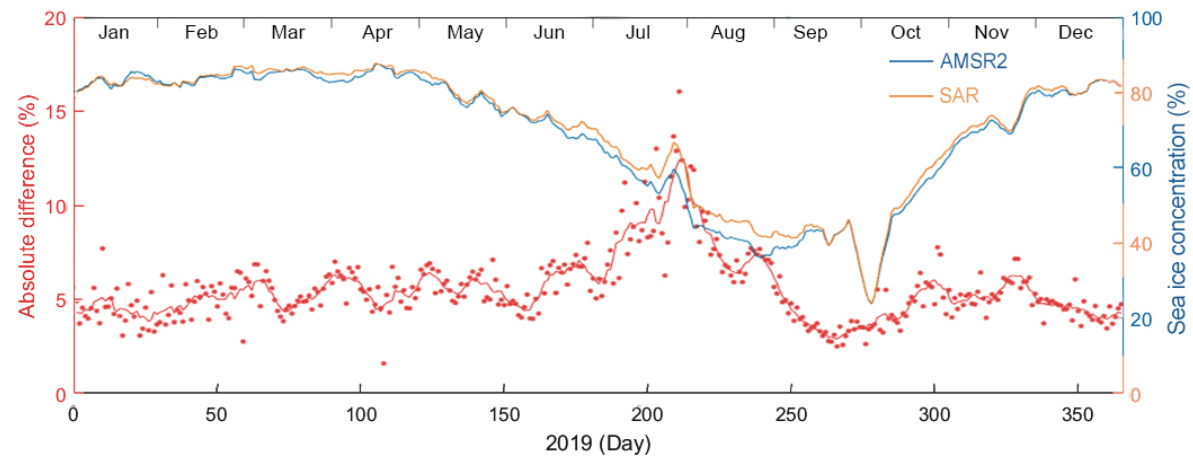

Figure 12. Comparison between the S1-derived Arctic sea ice concentration data and AMSR2 data for the whole year of 2019. The red dots reflect the absolute daily difference, and the red line is the $7 \mathrm{~d}$ average absolute difference. The blue line is the $7 \mathrm{~d}$ average sea ice concentration in the S1-covered area calculated based on the AMSR2 data, and the orange line is the $7 \mathrm{~d}$ average sea ice concentration calculated based on S1-derived results.

sis. Figure 11 shows an example of the S1-derived sea ice cover, the corresponding sea ice concentration, and a subsequent comparison with the AMSR2 data. This image was acquired from the Fram Strait and included large areas of both open water and floating sea ice. Figure 11a shows the RGB false-color composite image, and Fig. 11b is the corresponding sea ice cover result. Figure 11c presents the calculated sea ice concentration based on the S1-derived sea ice cover data, while the spatially collocated AMSR2 sea ice concentration data are shown in Fig. 11d. Figure 11e shows the differences in the sea ice concentration between AMSR2 and S1: red indicates the area where the S1-derived sea ice concentration is lower than the AMSR2 result, while blue indicates the opposite. Within the ice water mixing area in the northwest, the AMSR2 sea ice concentrations are overestimated, while in the ice water junction area, the concentrations are underestimated; this is the main reason for the absolute difference of $11.03 \%$ in this example.

For a more quantitative analysis, the daily absolute differences between the S1-derived and AMSR2 sea ice concentra- 


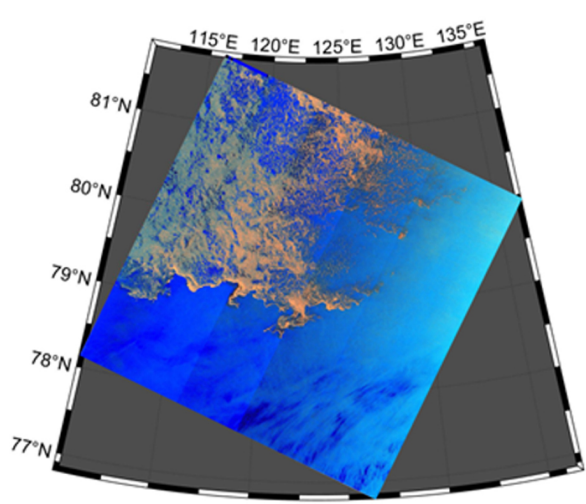

(a)

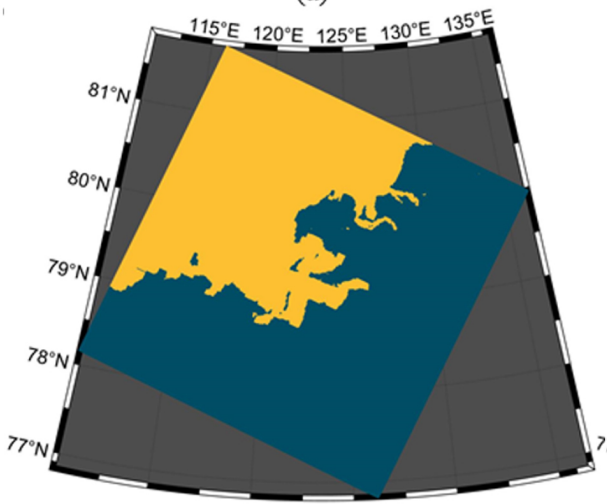

(c)

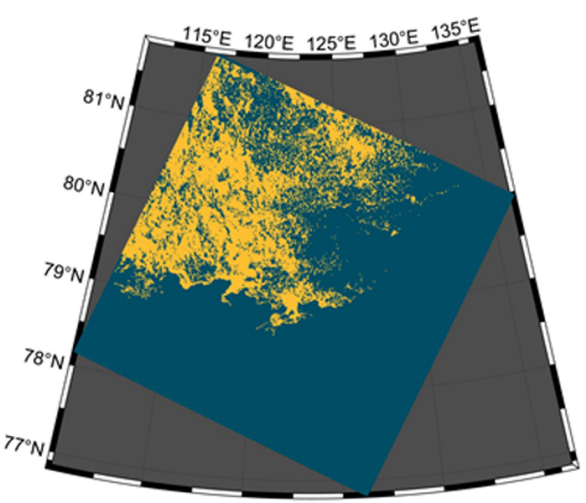

(b)

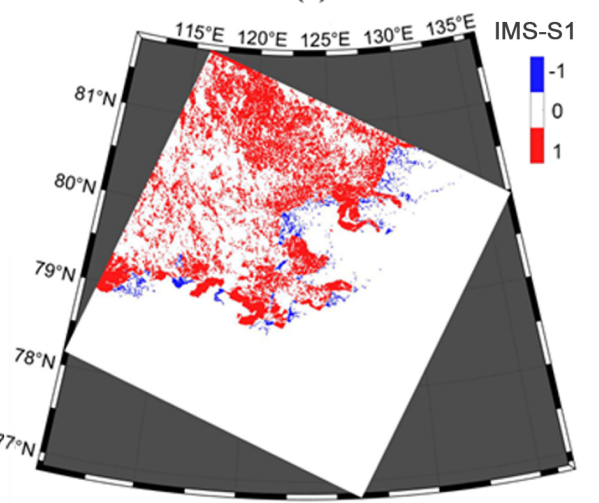

(d)

Figure 13. Example of S1-derived sea ice cover and its comparison with the IMS data. (a) RGB false-color composite image. Panels (b) and (c) are S1-derived sea ice cover and IMS sea ice cover, with yellow indicating sea ice and cyan indicating open water. (d) Differences in sea ice cover between the IMS and S1-derived results (IMS-S1). (Image ID: S1B_EW_GRDM_1SDH_20190822T232250_20190822T232354_017705_0214F8_D76F).

tion data are plotted in Fig. 12, with solid lines showing the $7 \mathrm{~d}$ moving average results. The sea ice concentration calculated by AMSR2 (in the region covered by S1 data) and S1derived results are plotted in blue and orange, respectively, in the form of $7 \mathrm{~d}$ moving average. During the summer season (June, July, and August), the sea ice concentration drops from $80 \%$ to approximately $40 \%$, and the absolute difference shows a noticeable increase to approximately $10 \%$; during the other seasons, the absolute difference is almost ubiquitously less than $6 \%$. For the whole year of 2019, the average absolute difference between the Arctic sea ice product derived from over $28000 \mathrm{~S} 1$ images and the AMSR2 data is $5.55 \%$. Research has demonstrated that sea ice concentration estimates from passive microwave observations are typically inaccurate in Arctic summer. This is due to the similar microwave radiation characteristics of sea ice and open water, which is mainly attributed to atmospheric effects (Han et al., 2018). Accordingly, the error of the AMSR 2 sea ice concentration data was estimated based on comparisons with (1) in situ ice observations, (2) ice concentration retrievals using other microwave algorithms, and (3) ice concentration data derived from higher-resolution optical sensors. The AMSR2 data show low errors at moderate and high ice concentrations (above $65 \%$ ), for which the error should not exceed $10 \%$. However, at low ice concentrations, the accuracy is low: the absolute error is $25 \%$ at $0 \%$ ice concentration and decreases for higher ice concentrations (Spreen et al., 2008). These errors are a plausible reason for the relatively large difference between the S1-derived sea ice concentration and AMSR2 data in the summer season, particularly in the period from middle July to the beginning of August.

\subsection{Comparison with the IMS sea ice cover data}

We also conducted a similar comparison between the S1derived Arctic sea ice cover data and the IMS sea ice cover data. The $400 \mathrm{~m}$ pixel size of the S1-derived sea ice cover data is comparable to that of the IMS data on a grid size of $1 \mathrm{~km}$. We directly compared the IMS data and S1-derived results by matching the nearest pixels of the two sets of data. Figure 13 shows an example of the S1-derived sea ice cover and its comparison with the IMS data in a vital area of the Northeast Passage in the Laptev Sea. Figure 13a shows the RGB false-color composite image of this example, and Fig. $13 \mathrm{~b}$ and $\mathrm{c}$ show the S1-derived sea ice cover and IMS 


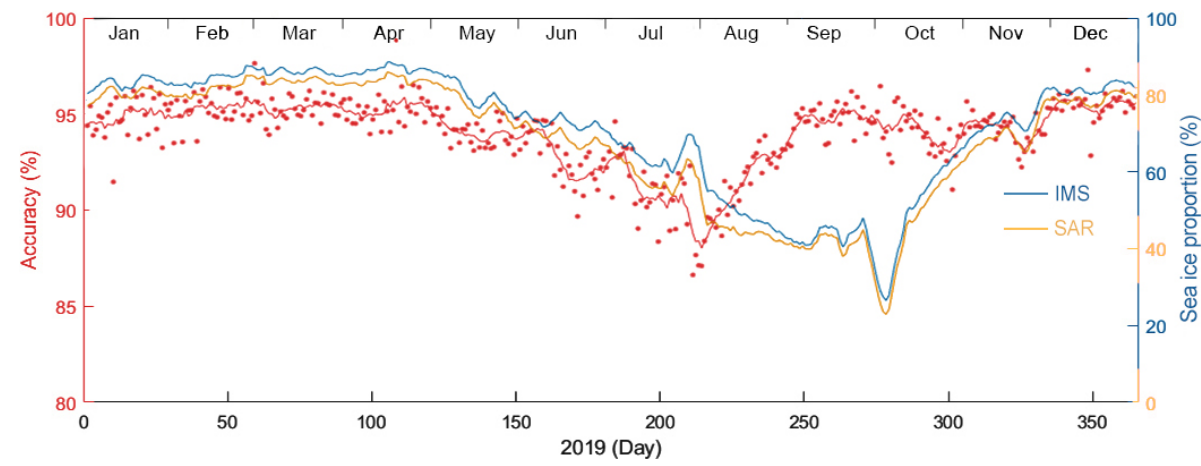

Figure 14. Comparison between the S1-derived Arctic sea ice cover product and the IMS data for the whole year of 2019. The red dots represent the daily average accuracy, and the red line is the $7 \mathrm{~d}$ average accuracy. The blue line is the $7 \mathrm{~d}$ average sea ice proportion in the S1-covered area calculated based on the AMSR2 data, and the orange line is the $7 \mathrm{~d}$ average sea ice proportion calculated based on S1-derived results.

data, respectively. Figure 13d shows the differences between the IMS and S1 results. As Fig. 13 shows, the S1-derived sea ice cover result is more detailed as the S1-derived outcome is acquired on a pixel-by-pixel basis, while the IMS data are smoothed, leading to the relatively low accuracy of $81.78 \%$ for this example.

The daily accuracy of the S1-derived sea ice cover compared with the IMS data is plotted in Fig. 14. For the whole year of 2019, the overall accuracy of the S1-derived Arctic sea ice data based on more than 28000 images is $93.98 \%$ compared with the IMS data. From January to April, the accuracy is approximately $95 \%$ with only minor fluctuations. From May onwards, the accuracy starts to decrease and reaches the lowest accuracy of $88 \%$ (according to the $7 \mathrm{~d}$ average result) at the end of July before quickly increasing to 95\% again through August. From September until the end of the year, the accuracy is approximately $95 \%$ with relatively large fluctuations. The sea ice proportion is also plotted in Fig. 14, representing the percentage of the sea ice area in the S1-covered region calculated based on the IMS data (in blue) and S1-derived results (in orange). Before July, the accuracy varies with a similar trend to the variation in the sea ice proportion. At the end of July, the sea ice proportion increases dramatically from approximately $60 \%$ to $70 \%$, and the corresponding comparison shows a minimum accuracy. Up to the end of September, although the sea ice proportion continues to decrease, the comparison suggests that the accuracy significantly increases. The variation in the accuracy is similar to the variation in the absolute difference achieved in comparison with the AMSR2 data. The discrepancy between the IMS data and the S1-derived results may be attributed to three aspects. (1) The IMS data are produced using data from various satellites with different spatial resolutions, and this may lead to smoother results in the data fusion process, whereas the S1-derived sea ice cover is pixel-based. This is especially obvious in areas with highly mixed sea ice and open water (e.g., the example shown in Fig. 13). (2) The
Table 2. List of variables and their descriptions in the NetCDF product.

\begin{tabular}{lll}
\hline No. & Variables & Descriptions \\
\hline 1 & Longitude & Longitude of each sea ice and land mask record \\
2 & Latitude & Latitude of each sea ice and land mask record \\
3 & SeaIce & 0 denotes open water, and 1 denotes sea ice \\
4 & Mask & 0 indicates no land, and 1 indicates land \\
\hline
\end{tabular}

daily IMS data are compiled based on various satellite observations within a day, whereas the S1-derived results are snapshots of the sea ice conditions at the time of SAR data acquisition. The temporal variation of sea ice can also lead to some differences. (3) Some error is caused by the limitations of the algorithm when deriving sea ice cover based on S1 and IMS data.

\section{Discussion}

In late July and early August, the S1-derived sea ice information shows a higher discrepancy between the AMSR2 sea ice concentration data and IMS data compared to the rest of the year. To further investigate this phenomenon, the discrepancies of the sea ice information derived from each scene of S1 data compared with the corresponding IMS and AMSR data are plotted in Fig. 16. The dots represent the center coordinates of the S1 EW images, and their colors represent the values of the absolute difference between the S1-derived sea ice concentration and the AMSR2 data (Fig. 16a) and the accuracy between the S1-derived results and IMS data (Fig. 16b). The time period of the comparison ranges from 25 July to 5 August 2019. Large discrepancies between the S1-derived results and the AMSR2 or IMS data are concentrated mainly in the East Siberian Sea and surrounding Greenland. During July and August, these two areas are mainly composed of extensive melting ice and large areas of brash ice. These 


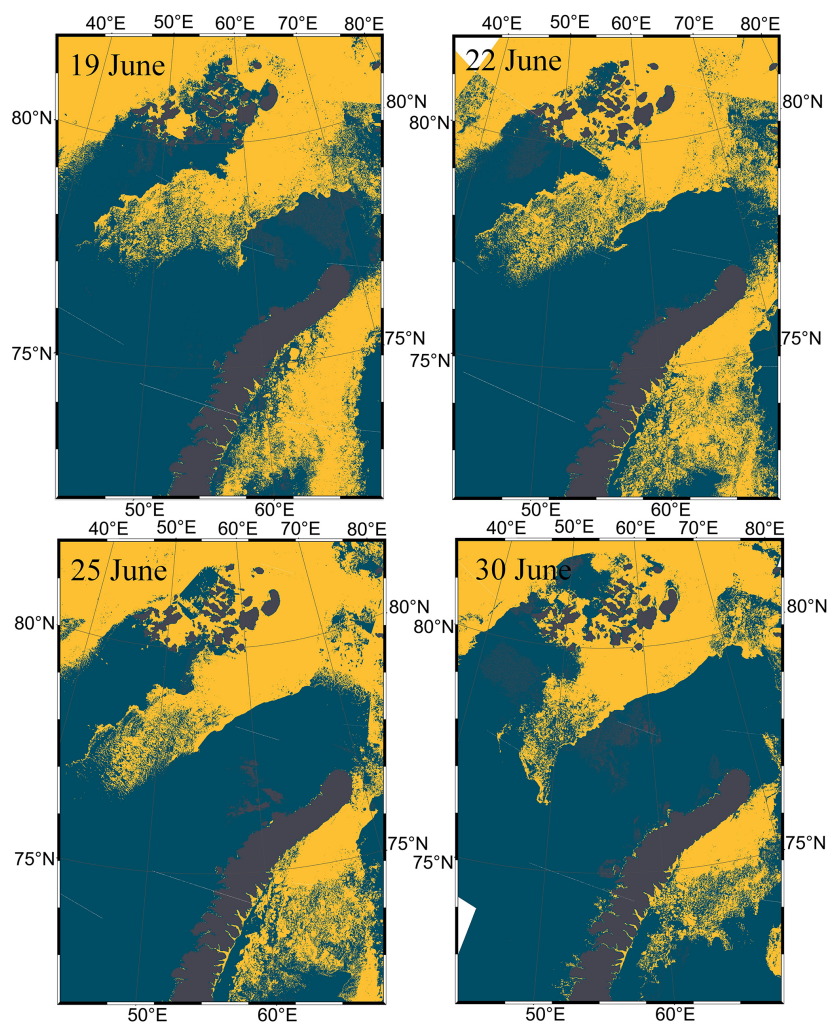

Figure 15. Example of the Arctic sea ice cover product in the Barents Sea and Kara Sea of the Northeast Passage. Yellow represents sea ice, cyan represents open water, and gray represents land.

complex ice conditions increase the difficulties of sea ice estimation by all methods. In the following, we will present two examples in the East Siberian Sea and on the Greenland coast for further demonstration.

Figure 17 shows an example of an image acquired on 29 July at $74^{\circ} \mathrm{N}, 163^{\circ} \mathrm{E}$ in the East Siberian Sea to illustrate the large discrepancies among the different data sources. Figure 17a presents the RGB false-color composite image, and panel (b) depicts the corresponding S1-derived sea ice cover result. The southeastern area of the S1-derived results shows that brash ice is overestimated. Figure $17 \mathrm{c}$ shows the IMS sea ice cover, which fails to detect the mixture of sea ice and open water and instead reveals continuous sea ice cover. Figure 17d shows the differences in the sea ice cover between the IMS and S1-derived results; the overall accuracy is $25.22 \%$ for this example. Figure $17 \mathrm{e}$ and $\mathrm{f}$ show the S1-derived sea ice concentration and the AMSR2 data, while panel $(\mathrm{g})$ shows the differences between them. Overestimation in the southeastern area of the S1-derived results leads to a high sea ice concentration, while the AMSR2 data show a much lower sea ice concentration than the S1-derived result, which causes a high average absolute difference of $28.06 \%$.

Figure 18 is the same as Fig. 17 but for the image acquired at $77^{\circ} \mathrm{N}, 11^{\circ} \mathrm{W}$ near northeastern Greenland. In this example, the S1-derived result preserves the details of sea ice cover in the MIZ. The IMS data still present the mixture of sea ice and open water as continuous ice, leading to an obvious overestimation and low overall accuracy of $61.56 \%$ (Fig. 18d). The relatively large absolute difference of $13.97 \%$ (Fig. 18g) between the S1-derived sea ice concentration and the AMSR2 data is due mainly to the AMSR2 data underestimating the pack ice in the northwest and slightly overestimating the brash ice in the northeast.

We found that the IMS data tend to overestimate sea ice cover by recording brash ice as a whole ice surface, which forfeits detailed features. This is likely because these data are obtained by implementing a daily average and smoothing the results based on various observations acquired at different times (as well as at different spatial resolutions) within a day. Furthermore, melted sea ice drifts significantly in the open sea during the summer season and leads to misclassification. We also found that the AMSR2 sea ice concentration data tend to underestimate pack sea ice near the coast in the summer season. We inferred that the changed radiometric characteristics of melted sea ice, particularly spatially extensive pack sea ice close to land, interfere with the radiometer-based retrieval of sea ice concentration. Thus, during July and August, the aggregation of brash ice and melted sea ice drifting along the coast of Greenland and within the East Siberian Sea causes high discrepancies among the S1-derived results and the AMSR2 and IMS data. In addition, because land occupies part of the S1 data, there are fewer valid areas involved in calculating the average discrepancy, which enlarges the proportion of local errors. This can also explain why the discrepancy increases with the increasing proportion of land in the $\mathrm{S} 1$ images.

In the process of deriving sea ice cover from the S1 data, we also found some examples of distinct misclassification, specifically for 208 cases in June, July, and August. The first typical type of such misclassification is caused mainly by a mismatch between the S1-imaged land and the GSHHG land masking data. Figure 19 shows such an example acquired in northern Greenland. Figure 19a and b show the S1 images in HV polarization without and with the land mask, respectively, clearly showing that part of the land imaged by S1 is not fully masked by the GSHHG data. As described in Sect. 3.3, we stretched the HV-polarized image by discarding $2 \%$ of the maximum and minimum values each. However, the extremely high values of the unmasked lands disturb the stretching process and cause information loss at low values, resulting in the misclassification results shown in Fig. 19c; thus, to reduce the influence of the high radar backscatter caused by unmasked land, we changed the threshold from $2 \%$ to $5 \%$. Figure $19 \mathrm{e}$ displays the corresponding correct classification result. After this modification for all 208 cases, 156 scenes of data present the correct sea ice segmentation results. These scenes were added to the comparisons with the IMS and AMSR2 data presented above.

Most of the remaining 52 cases (concentrated in the northern part of Greenland and Queen Elizabeth Islands; the S1B 

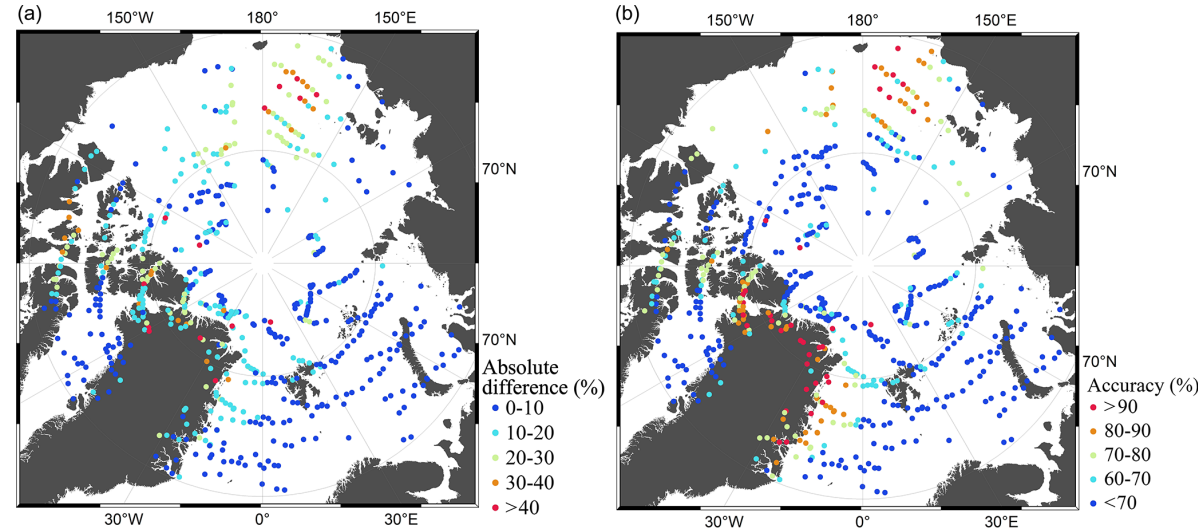

Figure 16. Plots of comparisons between the S1-derived sea ice cover and other data in the period from 25 July to 5 August 2019. (a) Absolute difference between the S1-derived sea ice concentration and the AMSR2 data. (b) Accuracy between the S1-derived sea ice cover results and the IMS data.

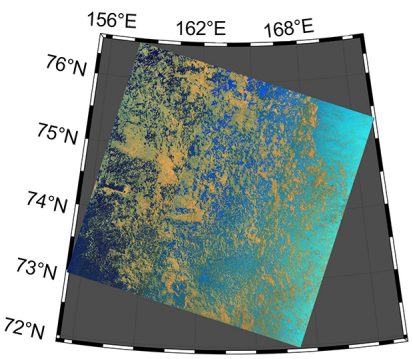

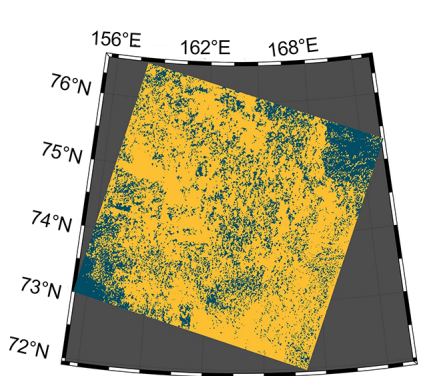

(b)

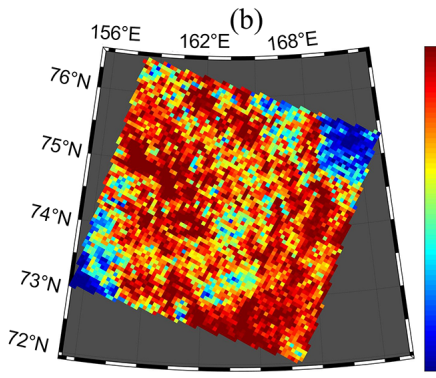

(e)

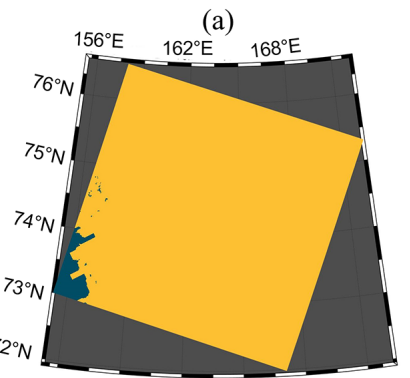

(c)

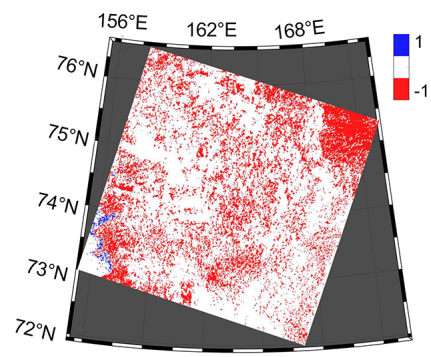

(d)

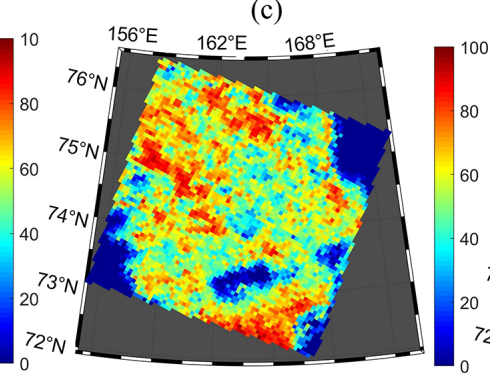

(f)

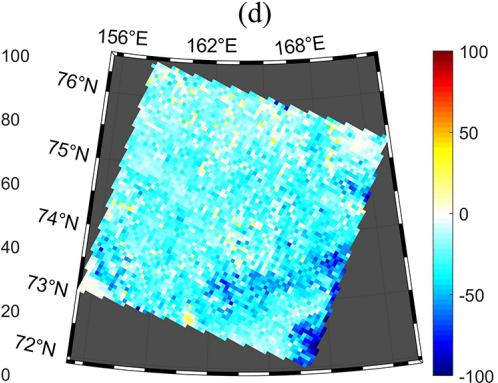

(g)

Figure 17. Example of the S1-derived sea ice cover acquired on 29 July and its comparison with the AMSR2 and IMS data. (a) RGB false-color composite image. Panels (b) and (c) are S1-derived sea ice cover and IMS sea ice cover, respectively. (d) Differences in the sea ice cover between the IMS and S1-derived results (IMS-S1). Panels (e) and (f) are S1-derived sea ice concentration and the AMSR2 data, respectively. (g) Differences in the sea ice concentration between the AMSR2 data and S1-derived results (AMSR2-S1). (Image ID: S1B_EW_GRDM_1SDH_20190729T200651_20190729T200751_017353_020A1F_94EB). 


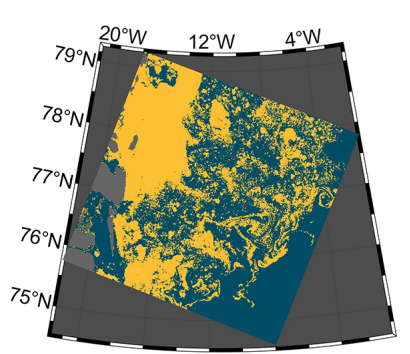

(b)

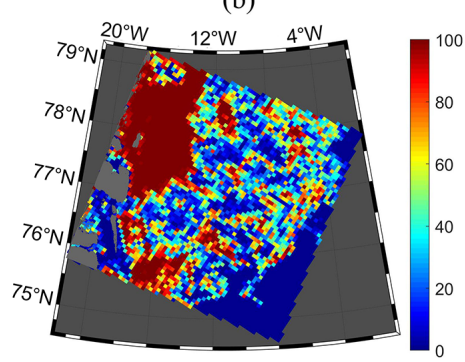

(e)

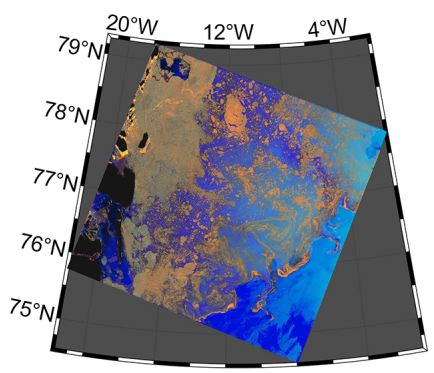

(a)

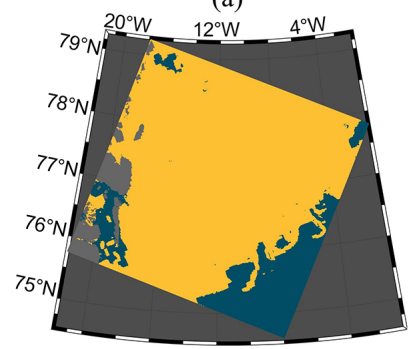

(c)

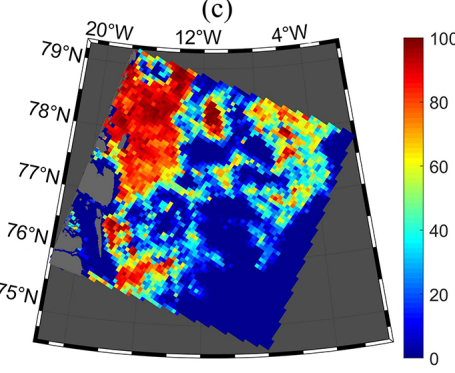

(f)

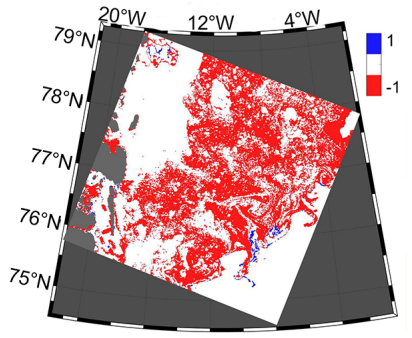

(d)

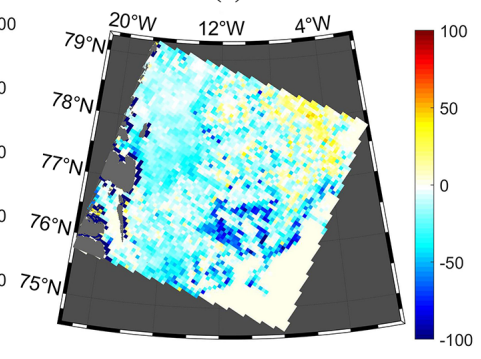

(g)

Figure 18. Same as Fig. 17 but for the photo acquired near Greenland. (Image ID: S1B_EW_GRDM_1SDH_20190802T080158_ 20190802T080258_017404_020BAA_48A5).

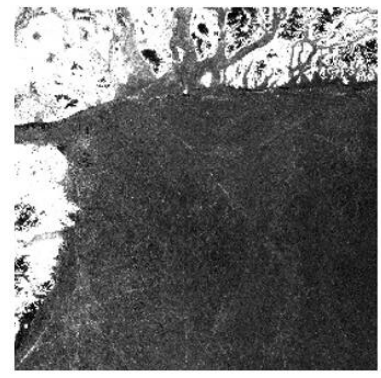

(a)

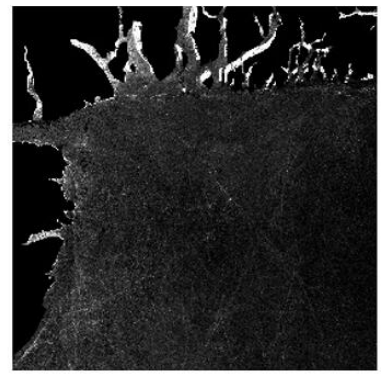

(b)

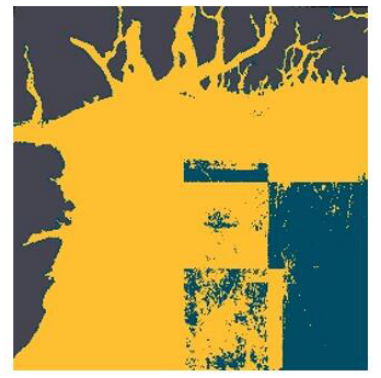

(c)

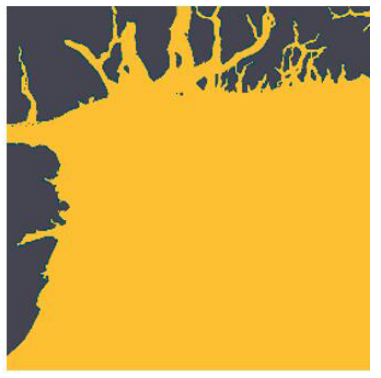

(d)

Figure 19. Example of a misclassification due to a mismatch between the S1-imaged land and GSHHG mask. (a) HV-polarized S1 image with no land mask. Panels (b) and (c) are HV-polarized S1 images with the land mask, stretched with a threshold of $2 \%$, and the corresponding misclassification result. Panels (d) and (e) are HV-polarized S1 images with the land mask, stretched with a threshold of $5 \%$, and the corresponding correct result. (Image ID: S1B_EW_GRDM_1SDH_20190701T185525_20190701T185629_016944_01FE25_A1F0).

data account for 42 of these cases) with distinct misclassifications show full sea ice cover except for land in the S1 images. Figure 20 shows two examples. The general impression of these two examples is that the sea ice radar backscatter exhibits significant spatial variation, even resembling a "jump" in the HV-polarized data (e.g., the first example). The accu- mulated water on the surface of melting sea ice can be inferred to trigger the dominant scattering mechanisms from volume scattering to surface scattering and therefore reduces the radar backscatter in $\mathrm{HV}$ polarization. Consequently, these areas are misclassified as open water. Notably, these 58 examples with distinct misclassifications were not included in 

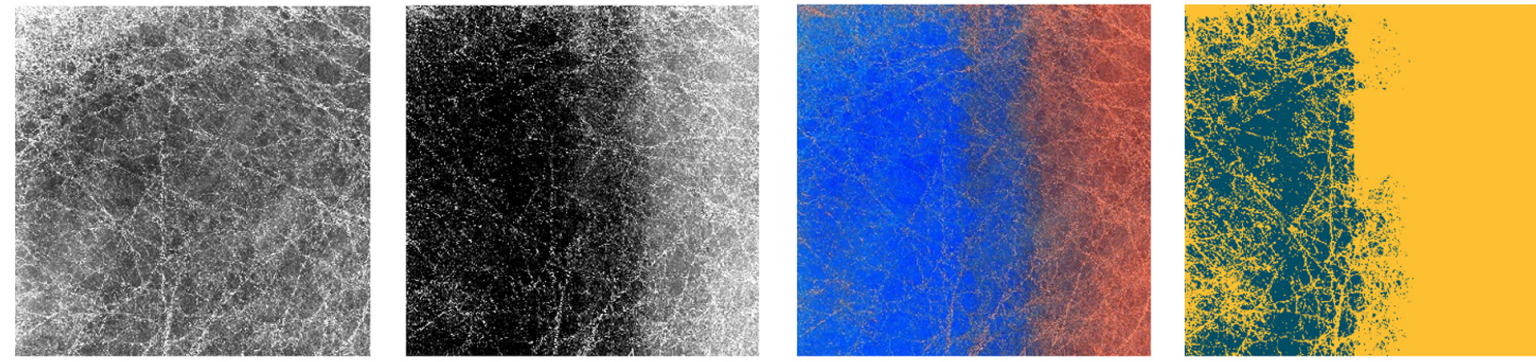

Image ID: S1B_EW_GRDM_1SDH_20190607T122033_20190607T122137_016590_01F3A2_2C14

Location: Northeast of Greenland. Center Longitude and Latitude: $38^{\circ} \mathrm{E}, 84^{\circ} \mathrm{N}$
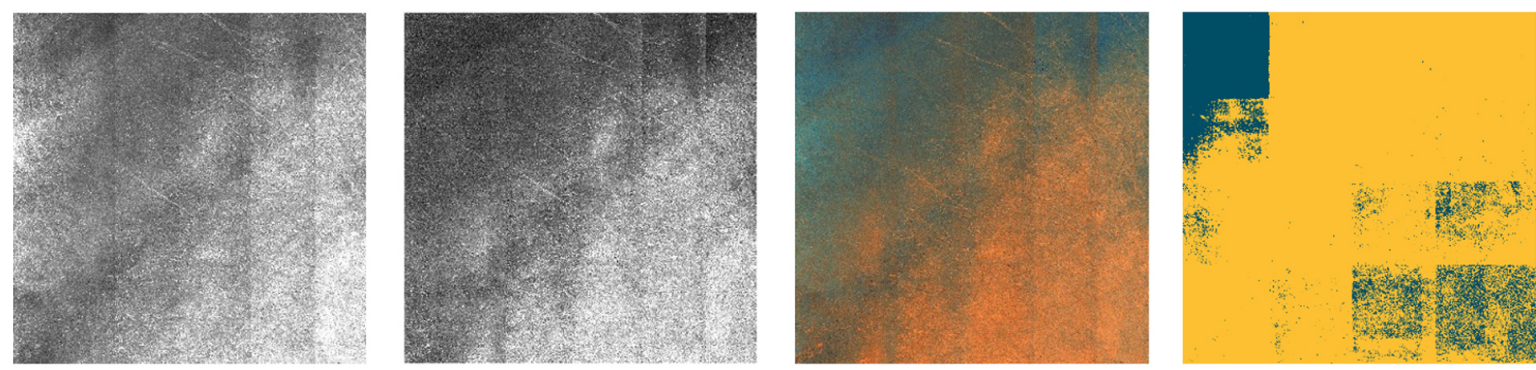

Image ID: SIE_S1B_EW_GRDM_1SDH_20190724T181612_20190724T181716_017279_0207FD_1D54

Location: North of the Queen Elizabeth Islands, Center Longitude and Latitude: $121^{\circ} \mathrm{W}, 85^{\circ} \mathrm{N}$

Figure 20. Examples of misclassifications that require further investigation. From left to right are S1 images in $\mathrm{HH}$ and $\mathrm{HV}$ polarization, RGB false-color composites and the S1-derived sea ice cover.

the comparisons with the IMS and AMSR2 data presented above.

\section{Data availability}

To facilitate related studies in the research community, the S1-derived sea ice data based on more than 28000 images are organized into an Arctic sea ice cover product in a standard format. The S1-derived Arctic sea ice cover product is stored in NetCDF-3 and follows the Climate and Forecast Metadata CF-1.7 convention (Eaton et al., 2014) to allow easy access from a range of standard tools across the leading computing platforms. Each record corresponds to the S1 images from which the Arctic sea ice cover product is derived. The product is named following the form SIC_ID_V1.0.nc, where SIC denotes sea ice cover, ID is the product ID of the $\mathrm{S} 1$ data, and V1.0 is the product version. Each record consists of four variables, namely, Longitude, Latitude, SeaIce, and Mask. The descriptions of the variables are listed in Table 2. Figure 15 shows an example of the Arctic sea ice cover product over a critical area, namely, the Northeast Passage, approximately located between 70 and $82^{\circ} \mathrm{N}, 40$ and $80^{\circ} \mathrm{E}$. Taking full advantage of SAR data, this sea ice cover product has high spatial and temporal resolutions and offers satisfactory coverage.

The S1 SAR data are downloaded from the Copernicus data hub (https://scihub.copernicus.eu/dhus/\#/home, last ac- cess: 10 June 2021, European Space Agency, 2021.). The authors would like to thank the European Space Agency (ESA) for providing the $\mathrm{S} 1$ images to users worldwide. The use of the reference AMSR2 data (https://seaice.uni-bremen. de/start/, last access: 10 June 2021, University of Bremen, 2021), IMS data (https://nsidc.org/data/G02156/versions/1, last access: 10 June 2021, U.S. National Ice Center, 2021), and GSHHG data (https://www.soest.hawaii.edu/pwessel/ gshhg/, last access: 10 June 2021, Wessel and Smith, 2021) is also acknowledged.

The developed Arctic sea ice cover data by S1 SAR data in 2019 are available at: https://doi.org/10.11922/sciencedb.00273 (Wang and $\mathrm{Li}, 2020$ ). More S1 SAR data are continuing to be processed since its launch, and the corresponding sea ice cover data in the Arctic will be also added to the repository.

\section{Summary and conclusion}

Due to the dramatic change in sea ice in polar regions, the most basic sea ice information, e.g., high-resolution sea ice cover data, is drawing increasing attention for scientific research, polar navigation, and offshore operations. While commonly used sea ice concentration data from spaceborne platforms are generally provided at spatial resolutions ranging from dozens of kilometers to several kilometers, spaceborne SAR can provide such information at a spatial res- 
olution of a few hundred meters or even higher. Since the launch of S1A in 2014 and S1B in 2016, these two satellites have routinely acquired SAR data in polar regions, and they are expected to continue operating for more than 10 years, similar to ERS/SAR and ENVISAT/ASAR. Therefore, S1derived sea ice data with a high spatial resolution and high accuracy can offer great support for better understanding the ongoing changes in polar regions. Thus, in this study, we aimed to develop a robust method of deriving sea ice cover data in the Arctic by S1A and S1B and also attempted to generate an easily accessed and handled product for other usage.

The proposed sea ice segmentation model is built upon the CNN architecture of U-Net, which can provide pixel-level segmentation. When applying deep learning in the field of remote sensing, the lack of labeled data constitutes a major obstacle. Thus, U-Net, which was designed for small training samples, is particularly suitable for the sea ice segmentation task. In addition, the augmentation process dramatically increases both the quantity and the diversity of the training samples and further guarantees robust performance against overfitting even when only limited labeled data are available. Although HV-polarized SAR data are particularly suitable for sea ice detection, previous studies have suggested that combined information from both $\mathrm{HH}$ and $\mathrm{HV}$ channels can benefit this task. Therefore, we synthesized S1 HV-polarized data with polarization difference and polarization ratio data between $\mathrm{HH}$ and $\mathrm{HV}$ polarization into an RGB false-color composite as the input for the U-Net model. Sea ice features in SAR images vary significantly. For instance, different types of sea ice can present variable radar backscatter characteristics, as can the same type of sea ice in different seasons. On the other hand, open water in the MIZ can also alert radar backscatter due to changes in the sea surface wind field. Therefore, it is difficult to train a single U-Net model to address various sea ice and open water states. Our solution is to train different U-Net models to obtain five classifiers with diverse specializations. These five U-Net classifiers are combined by an integrated stacking model to generate an aggregate sea ice classifier. The combination enables every single model to fully utilize its strengths and mitigate its weaknesses, resulting in higher accuracy and sensitivity. Eventually, we applied the proposed model to more than $28000 \mathrm{~S} 1$ images acquired in 2019. Consequently, we generated a sea ice cover product with a spatial resolution of $400 \mathrm{~m}$.

We conducted a comparison between the S1-derived sea ice cover and the pixel-level visual interpretation results over 96 cases. For the cases with sea ice proportion less than $25 \%$ or larger than $50 \%$, the accuracy reaches $\sim 99 \%$. The overall accuracy of the 96 cases is $96.10 \%$. Case-by-case competition for all the Arctic sea ice cover data derived from more than $28000 \mathrm{~S} 1$ images have also been conducted. We compared the S1-derived sea ice cover data with AMSR2 sea ice concentration data and IMS sea ice cover data. The average absolute difference between the S1-derived and AMSR2derived sea ice concentrations is $5.55 \%$, and the overall ac- curacy of the S1-derived sea ice cover data is $93.98 \%$ compared with the IMS data. Although this overall accuracy is promising, both comparisons reveal obvious seasonal fluctuations, particularly in the summer months of July and August. The melting of sea ice in summer is a major contributor to the large discrepancy between the S1 results and the AMSR2 data (noting that the IMS data also include information from radiometer data). Melting sea ice not only has great impacts on microwave radiation, which consequently induces retrieval errors by radiometer data, but also alert radar backscatter; as a result, the trained model may misclassify sea ice and open water. On the other hand, in the summer season, along with melting, thin sea ice tends to mix highly with open water, and this mixture may present radar backscatter characteristics similar to those of open water; thus, the model will overestimate the sea ice area. Nevertheless, the complex situation of sea ice in summer presents a challenge for deriving sea ice cover, and more efforts should be paid to this effect in the future.

In this study, we conducted research constituting an important step in routinely generating SAR-derived sea ice cover products. More historical S1 images are being processed, and more comparisons with other datasets will be conducted. We hope that the developed high-resolution SAR-derived Arctic sea ice cover product can be utilized for different purposes by different communities and can further enhance scientific research, environmental protection, and resource utilization in the Arctic.

Author contributions. XML conceived the idea and designed the research. YRW developed the method and carried out the analysis. XML and YRW wrote the manuscript together.

Competing interests. The authors declare that they have no conflict of interest.

Financial support. This research has been supported by the Ministry of Science and Technology of the People's Republic of China (grant nos. NSFC-42025605 and 2018YFC1407100).

Review statement. This paper was edited by Prasad Gogineni and reviewed by two anonymous referees.

\section{References}

Aldenhoff, W., Heuzé, C., and Eriksson, L. E.: Sensitivity of Radar Altimeter Waveform to Changes in Sea Ice Type at Resolution of Synthetic Aperture Radar, Remote Sensing, 11, 2602, https://doi.org/10.3390/rs11222602, 2019.

Boulze, H., Korosov, A., and Brajard, J.: Classification of sea ice types in Sentinel-1 SAR data using con- 
volutional neural networks, Remote Sensing, 12, 2165, https://doi.org/10.3390/rs12132165, 2020.

Clausi, D. A.: Comparison and fusion of co-occurrence, Gabor and MRF texture features for classification of SAR sea-ice imagery, Atmos.-Ocean, 39, 183-194, https://doi.org/10.1080/07055900, 2001

Clausi, D. A. and Zhao, Y.: Grey level co-occurrence integrated algorithm (GLCIA): a superior computational method to rapidly determine co-occurrence probability texture features, Comput. Geosci., 29, 837-850, https://doi.org/10.1016/S00983004(03)00089-X, 2003.

Comiso, J. and Sullivan, C.: Satellite microwave and in situ observations of the Weddell Sea ice cover and its marginal ice zone, J. Geophys. Res.-Oceans, 91, 9663-9681, https://doi.org/10.1029/jc091ic08p09663, 1986.

Comiso, J. C., Cavalieri, D. J., Parkinson, C. L., and Gloersen, P.: Passive microwave algorithms for sea ice concentration: A comparison of two techniques, Remote Sens. Environ., 60, 357-384, https://doi.org/10.1016/s0034-4257(96)00220-9, 1997.

Dong, J., Xiao, X., Sheldon, S., Biradar, C., Duong, N. D., and Hazarika, M.: A comparison of forest cover maps in Mainland Southeast Asia from multiple sources: PALSAR, MERIS, MODIS and FRA, Remote Sens. Environ., 127, 60-73, https://doi.org/10.1016/j.rse.2012.08.022, 2012.

Dierking, W.: Sea ice monitoring by synthetic aperture radar, Oceanography, 26, 100-111, 2013.

Eaton, B., Gregory, J., Drach, B., Taylor, K., Hankin, S., Blower, J., Caron, J., Signell, R., Bentley, P., Rappa, G., Höck, H., Pamment, A., Juckes, M., Walsh, A., and Graybeal, J.: NetCDF Climate and Forecast (CF) Metadata Conventions: Version 1.7.2, available at: http://cfconventions.org/Data/cf-conventions/cf-conventions- 1 . 7/build/cf-conventions.pdf (last access: 10 June 2021), 2014.

European Space Agency: Sentinel-1 (S1), available at: https:// scihub.copernicus.eu/dhus/\#/home, last access: 10 June 2021.

Frost, A., Jacobsen, S., and Singha, S.: High resolution sea ice drift estimation using combined TerraSAR-X and RADARSAT2 data: First tests, 2017 IEEE International Geoscience and Remote Sensing Symposium (IGARSS), Fort Worth, Texas, USA, 23-28 July 2017, 342-345, 2017.

Gloersen, P., Campbell, W. J., Cavalieri, D. J., Comiso, J. C., Parkinson, C. L., and Zwally, H. J.: Satellite passive microwave observations and analysis of Arctic and Antarctic sea ice, 1978-1987, Ann. Glaciol., 17, 149-154, https://doi.org/10.3189/s0260305500012751, 1993.

Han, H., Lee, S., and Kim, H.-C.: Estimation of summer sea ice concentration in the Chukchi Sea using AMSR2 observation and numerical weather prediction data, in: 20th EGU General Assembly, Vienna, Austria, 4-13 April 2018, 6485, 2018.

Hersbach, H., Bell, B., Berrisford, P., Hirahara, S., Horányi, A., Muñoz-Sabater, J., Nicolas, J., Peubey, C., Radu, R., and Schepers, D.: The ERA5 global reanalysis, Q. J. Roy. Meteor. Soc., 146, 1999-2049, https://doi.org/10.1002/qj.3803, 2020.

Hollinger, J. P., Peirce, J. L., and Poe, G. A.: SSM/I instrument evaluation, IEEE T. Geosci. Remote, 28, 781-790, https://doi.org/10.1109/36.58964, 1990.

Hong, D.-B. and Yang, C.-S.: Automatic discrimination approach of sea ice in the Arctic Ocean using Sentinel-1 Extra Wide Swath dual-polarized SAR data, Int. J. Remote Sens., 39, 4469-4483, https://doi.org/10.1080/01431161.2017.1415486, 2018.
Karvonen, J.: Baltic sea ice concentration estimation based on Cband dual-polarized SAR data, IEEE T. Geosci. Remote, 52, 5558-5566, https://doi.org/10.1109/TGRS.2013.2290331, 2013.

Kingma, D. P., and Ba, J.: Adam: A method for stochastic optimization, arXiv [preprint], arXiv:1412.6980, 22 December 2014.

Komarov, A. S. and Buehner, M.: Automated detection of ice and open water from dual-polarization RADARSAT-2 images for data assimilation, IEEE T. Geosci. Remote, 55, 5755-5769, https://doi.org/10.1109/TGRS.2017.2713987, 2017.

Kunkee, D. B., Poe, G. A., Boucher, D. J., Swadley, S. D., Hong, Y., Wessel, J. E., and Uliana, E. A.: Design and evaluation of the first special sensor microwave imager/sounder, IEEE T. Geosci. Remote, 46, 863-883, https://doi.org/10.1109/TGRS.2008.917980, 2008.

Kwok, R. and Rothrock, D.: Decline in Arctic sea ice thickness from submarine and ICESat records: 1958-2008, Geophys. Res. Lett., 36, https://doi.org/10.1029/2009GL039035, 2009.

Leigh, S., Wang, Z., and Clausi, D. A.: Automated icewater classification using dual polarization SAR satellite imagery, IEEE T. Geosci. Remote, 52, 5529-5539, https://doi.org/10.1109/TGRS.2013.2290231, 2013.

Li, X.-M., Sun, Y., and Zhang, Q.: Extraction of Sea Ice Cover by Sentinel-1 SAR Based on Support Vector Machine With Unsupervised Generation of Training Data, IEEE T. Geosci. Remote, 59, 3040-3053, https://doi.org/10.1109/TGRS.2020.3007789, 2020.

Liu, H., Guo, H., and Zhang, L.: SVM-based sea ice classification using textural features and concentration from RADARSAT-2 dual-pol ScanSAR data, IEEE J. Sel. Top. Appl., 8, 1601-1613, https://doi.org/10.1109/JSTARS.2014.2365215, 2014.

Murashkin, D., Spreen, G., Huntemann, M., and Dierking, W.: Method for detection of leads from Sentinel-1 SAR images, Ann Glaciol., 59, 124-136, https://doi.org/10.1017/aog.2018.6, 2018.

Nghiem, S., Rigor, I., Perovich, D., Clemente-Colón, P., Weatherly, J., and Neumann, G.: Rapid reduction of Arctic perennial sea ice, Geophys. Res. Lett., 34, L19504, https://doi.org/10.1029/2007GL031138, 2007.

Onarheim, I. H., Eldevik, T., Smedsrud, L. H., and Stroeve, J. C.: Seasonal and regional manifestation of Arctic sea ice loss, J. Climate, 31, 4917-4932, https://doi.org/10.1175/JCLI-D-170427.1, 2018.

Pachauri, R. K., Allen, M. R., Barros, V. R., Broome, J., Cramer, W., Christ, R., Church, J. A., Clarke, L., Dahe, Q., and Dasgupta, P.: Climate change 2014: synthesis report, in: Contribution of Working Groups I, II and III to the fifth assessment report of the Intergovernmental Panel on Climate Change, edited by: Pachauri, R. and Meyer, L., IPCC, Geneva, Switzerland, 151 pp., available at: http://www.ipcc.ch/publicationsanddata/ publicationsipccfourthassessmentreportsynthesisreport.htm (last access: 10 June 2021), 2014.

Park, J.-W., Korosov, A. A., Babiker, M., Sandven, S., and Won, J.-S.: Efficient thermal noise removal for Sentinel-1 TOPSAR cross-polarization channel, IEEE T. Geosci. Remote, 56, 15551565, https://doi.org/10.1109/TGRS.2017.2765248, 2017.

Parkinson, C. L., Cavalieri, D. J., Gloersen, P., Zwally, H. J., and Comiso, J. C.: Arctic sea ice extents, areas, and trends, 1978-1996, J. Geophys. Res.-Oceans, 104, 20837 20856, https://doi.org/10.1029/1999jc900082, 1999. 
Power, D., Youden, J., Lane, K., Randell, C., and Flett, D.: Iceberg detection capabilities of RADARSAT synthetic aperture radar, Can. J. Remote Sens., 27, 476-486, https://doi.org/10.1080/07038992.2001.10854888, 2001.

Rampal, P., Weiss, J., and Marsan, D.: Positive trend in the mean speed and deformation rate of Arctic sea ice, 1979-2007, J. Geophys. Res.-Oceans, 114, C05013, https://doi.org/10.1029/2008jc005066, 2009.

Ramsay, B. H.: The interactive multisensor snow and ice mapping system, Hydrol. Proc., 12, 1537-1546, https://doi.org/10.1002/(sici)10991085(199808/09)12:10/11<1537::aid-hyp679>3.0.co;2-a, 1998.

Ressel, R., Frost, A., and Lehner, S.: A neural networkbased classification for sea ice types on X-band SAR images, IEEE J. Sel. Top. Appl., 8, 3672-3680, https://doi.org/10.1109/jstars.2015.2436993. 2015.

Ronneberger, O., Fischer, P., and Brox, T.: U-net: Convolutional networks for biomedical image segmentation, Medical Image Computing and Computer-Assisted Intervention MICCAI 2015, Springer International Publishing, 234-241, https://doi.org/10.1007/978-3-319-24574-4_28, 2015.

Scheuchl, B., Flett, D., Caves, R., and Cumming, I.: Potential of RADARSAT-2 data for operational sea ice monitoring, Can. J. Remote Sens., 30, 448-461, https://doi.org/10.5589/m04-011, 2004.

Serreze, M. C. and Barry, R. G.: Processes and impacts of Arctic amplification: A research synthesis, Global Planet. Change, 77, 85-96, https://doi.org/10.1016/j.gloplacha.2011.03.004, 2011.

Soh, L.-K. and Tsatsoulis, C.: Texture analysis of SAR sea ice imagery using gray level co-occurrence matrices, IEEE T. Geosci. Remote, 37, 780-795, https://doi.org/10.1109/36.752194, 1999.

Spreen, G., Kaleschke, L., and Heygster, G.: Operational sea ice remote sensing with AMSR-E $89 \mathrm{GHz}$ channels, Proceedings of the 2005 IEEE International Geoscience and Remote Sensing Symposium, Int. Geosci. Remote Se., 4033-4036, 2005.

Spreen, G., Kaleschke, L., and Heygster, G.: Sea ice remote sensing using AMSR-E 89-GHz channels, J. Geophys. Res.-Oceans, 113, C02S03, https://doi.org/10.1029/2005jc003384, 2008.

Stroeve, J., Serreze, M., Fetterer, F., Arbetter, T., Meier, W., Maslanik, J., and Knowles, K.: Tracking the Arctic's shrinking ice cover: Another extreme September minimum in 2004, Geophys. Res. Lett., 32, L04501, https://doi.org/10.1029/2004gl021810, 2005.
Strong, C. and Rigor, I. G.: Arctic marginal ice zone trending wider in summer and narrower in winter, Geophys. Res. Lett., 40, 4864-4868, https://doi.org/10.1002/grl.50928, 2013.

Sun, Y. and Li, X.-M.: Denoising Sentinel-1 ExtraWide Mode Cross-polarization Images over Sea Ice, IEEE T. Geosci. Remote, 59, 2116-2131, https://doi.org/10.1109/TGRS.2020.3005831, 2020.

Tan, W., Li, J., Xu, L., and Chapman, M. A.: Semiautomated segmentation of Sentinel-1 SAR imagery for mapping sea ice in Labrador coast, IEEE J. Sel. Top. Appl., 11, 1419-1432, https://doi.org/10.1109/jstars.2018.2806640, 2018.

Thomson, J. and Rogers, W. E.: Swell and sea in the emerging Arctic Ocean, Geophys. Res. Lett., 41, 3136-3140, https://doi.org/10.1002/2014g1059983, 2014.

University of Bremen: Advanced Microwave Scanning Radiometer 2 (AMSR2), available at: https://seaice.uni-bremen.de/start/, last access: 10 June 2021.

U.S. National Ice Center (USNIC): Interactive Multisensor Snow and Ice Mapping System (IMS), available at: https://nsidc.org/ data/G02156/versions/1, last access: 10 June 2021.

Wang, J., Duguay, C. R., Clausi, D. A., Pinard, V., and Howell, S. E.: Semi-automated classification of lake ice cover using dual polarization RADARSAT-2 imagery, Remote Sensing, 10, 1727, https://doi.org/10.3390/rs10111727, 2018.

Wang, Y. and Li, X.: Arctic sea ice cover product based on spaceborne synthetic aperture radar, V1 [dataset], Science Data Bank, https://doi.org/10.11922/sciencedb.00273, 2020.

Wessel, P. and Smith, W. H. F.: Global Self-consistent Hierarchical High-resolution Geography Database (GSHHG), available at: https://www.soest.hawaii.edu/pwessel/gshhg/, last access: 10 June 2021.

Wolpert, D. H.: Stacked generalization, Neural networks, 5, 241259, https://doi.org/10.1016/S0893-6080(05)80023-1, 1992.

Wolpert, D. H. and Macready, W. G.: No free lunch theorems for optimization, IEEE T. Evolut. Comput., 1, 67-82, https://doi.org/10.1109/4235.585893, 1997.

Zakhvatkina, N., Korosov, A., Muckenhuber, S., Sandven, S., and Babiker, M.: Operational algorithm for ice-water classification on dual-polarized RADARSAT-2 images, The Cryosphere, 11, 33-46, https://doi.org/10.5194/tc-11-33-2017, 2017. 